\title{
Normalization of glucose metabolism by Exendin- 4 in the chronic phase after stroke promotes functional recovery in diabetes
}

Ingrid Augestad

Karolinska Institutet

Doortje Dekens

Karolinska Institutet

Dimitra Karampatsi

Karolinska Institutet

Osama Elabi

Lund University: Lunds Universitet

Alexander Zabala

Karolinska Institutet

Hiranya Pintana

Karolinska Institutet

Martin Larsson

Karolinska Institutet

Thomas Nyström

Karolinska Institutet

Gesine Paul

Lund University: Lunds Universitet

Vladimer Darsalia

Karolinska Institute: Karolinska Institutet

Cesare Patrone ( $\nabla$ cesare.patrone@ki.se )

Karolinska Institutet https://orcid.org/0000-0003-0470-4606

Original investigation

Keywords: T2D, Stroke, Neurological recovery, Parvalbumin, vascular remodeling, fibrotic scar, DPP-4 inhibitors, sulfonylureas

Posted Date: February 23rd, 2021

DOI: https://doi.org/10.21203/rs.3.rs-210113/v1 
License: (c) (i) This work is licensed under a Creative Commons Attribution 4.0 International License. Read Full License 


\section{Abstract \\ Background}

Glucagon-like peptide-1 receptor (GLP-1R) activation can decrease stroke risk in people with type 2 diabetes (T2D). Moreover, animal studies have shown the efficacy of GLP-1R agonists to counteract stroke-induced acute brain damage. Whether GLP-1R activation can also improve stroke recovery during the post-acute, chronic phase after stroke, however, remains to be determined. We investigated whether post-acute, chronic administration of the GLP-1R agonist Exendin-4 improves poststroke recovery and examined possible underlying mechanisms in T2D and non-T2D mice.

\section{Methods}

We induced stroke via transient middle cerebral artery occlusion (tMCAO) in T2D/obese mice (8 months of high-fat diet) and age-matched controls. Exendin- 4 was administered daily for 8 weeks from day 3 after $\mathrm{tMCAO}$. We assessed functional recovery by weekly upper-limb grip strength tests, while insulin sensitivity and glycemia were evaluated at 4 and 8 weeks after tMCAO. Neuronal cell death, strokeinduced neurogenesis, neuroinflammation, potential atrophy of GABAergic, parvalbumin + interneurons, poststroke vascular remodeling and fibrotic scar formation were investigated by immunohistochemistry.

\section{Results}

Exendin-4 entirely normalized the T2D-induced impairment of forepaw grip strength recovery. The recovery correlated with the normalization of glycemia and insulin sensitivity. We also show that Exendin4 counteracted the T2D-induced atrophy of parvalbumin + interneurons and decreased microglia activation. In addition, Exendin-4 normalized density and pericyte coverage of microvessels and restored fibrotic scar formation in T2D mice. In non-T2D mice the recovery effect of Exendin-4 was minor.

\section{Conclusion}

This study demonstrates that post-acute, chronic GLP-1R activation mediates neurological recovery after stroke in T2D mice likely through the normalization of glucose metabolism and neuroplasticity mechanisms as well as improved vascular remodeling in the recovery phase. The results promote launching clinical trials investigating whether GLP-1R agonists improve the efficacy of rehabilitation after stroke in people with T2D.

\section{Background}

Glucagon-like peptide-1 receptor (GLP-1R) agonists are glucose-lowering drugs for type 2 diabetes (T2D) that act in a glucose-dependent manner, with minimal risks of hypoglycemia (1-3). Large randomized 
clinical trials have shown that these drugs can also reduce the risk of major cardiovascular events (411). Moreover, several preclinical studies using experimental stroke models indicate that GLP-1R agonists can reduce stroke-induced brain damage when given acutely or sub-acutely before stroke, at stroke onset or immediately after stroke, in both normal or diabetic rodents (12-14). What remains to be determined is whether the chronic activation of GLP-1R in the post-acute phase of stroke is efficacious to improve functional recovery. This knowledge is crucial since T2D is a strong risk factor for stroke (15-17) and it is estimated that by 2030 the number of people with diabetes will reach 439 million (18). Therefore, people with T2D constitute an enormous candidate group not only for stroke prevention but also for stroke treatment/care. Moreover, T2D worsens initial stroke outcome and it is also a strong predictor of poststroke dependency on supportive care in activities of daily living (19-22). Thus, new and efficacious poststroke pharmacological treatments in people with T2D are highly needed.

Acute (23) and persistent (up-to 72 hours (24)) hyperglycemia after stroke is common in both normal and diabetic individuals and is associated with worse outcome. The effective achievement of normoglycemia is considered to be beneficial in the acute setting, but conclusive evidence is still lacking because aggressive glucose control can be complicated by hypoglycemic episodes that are detrimental to stroke outcome (25). Importantly, the potential efficacy of glycemia regulation in the post-acute, chronic phase after stroke to improve recovery has not been studied clinically. We recently showed the potential efficacy of this strategy by using both dipeptidyl peptidase-4 (DPP-4) inhibition and sulfonylurea treatment (26). Whether GLP-1 analogues also can improve neurological recovery after stroke remains to be addressed.

There are several mechanisms through which delayed GLP-1R activation after stroke could improve recovery in diabetes. For instance, GLP-1 improves endothelial function in T2D $(27,28)$ and GLP-1R activation after stroke can stimulate angiogenesis $(29,30)$. It has been also shown that the GLP-1R agonist Exendin-4 can stabilize the blood-brain barrier (BBB) after stroke and reduce inflammation (3133). Moreover, the modulation of GABAergic activity plays a central role in facilitating functional recovery after stroke (34) and recently it has been shown that GLP-1R activation can modulate GABA signaling (35) and affects specific populations of GABAergic interneurons $(36,37)$. Finally, stroke-induced neurogenesis from the neural stem cells (NSC) in the subventricular zone (SVZ) has been suggested to contribute to stroke recovery (38) and GLP-1R agonists do enhance this process in rodent stroke models $(39,40)$. Whether these or additional mechanisms triggered by GLP-1R activation improve recovery in the chronic phase after stroke remains to be determined. It also remains to be investigated whether these positive effects occur through normalization of glucose metabolism (hyperglycemia and insulin resistance), since many of the above-mentioned pre-clinical studies have used non-diabetic rodents.

The first aim of this study was to determine whether a delayed treatment after stroke (starting 3 days post-stroke) with the specific GLP-1R agonist Exendin-4 (Ex-4) (41) improves neurological recovery in a clinically-relevant mouse model of obesity-induced T2D $(26,42)$. We also investigated whether neurological recovery was associated with improved glucose metabolism during the recovery phase. Finally, we investigated whether GLP-1R activation enhanced stroke-induced neurogenesis, modulated neuroinflammation and GABAergic interneurons, and impacted on vascular remodeling and fibrotic scar 
formation in the peri-infarct/infarct regions. To investigate whether the potential efficacy of GLP-1R activation in stroke recovery was due to the improvement of diabetic pathology, we performed a parallel study in age-matched non-diabetic mice.

\section{Methods}

\section{The T2D animal model and experimental design}

All applicable international, national, and/or institutional guidelines for the care and use of animals were followed where the studies were conducted under the ethical approval ID1126 (Karolinska Institutet).

Study 1 (diabetic study): Thirty male C57BL/6JRj mice (Janvier Labs, France) were used in this study. All mice were housed in environmentally controlled conditions $\left(25 \pm 0.5^{\circ} \mathrm{C}, 12 / 12\right.$ hours light/dark cycle with ad libitum access to food and water). From 4 weeks of age, the mice were fed with either standard laboratory diet (SD) ( $n=10)$ or high fat diet (HFD: 60\% energy from fat) $(n=20)$ for 8 months. To verify induced T2D after HFD feeding, body weight (BW), fasting blood glucose and insulin sensitivity were measured. Once obesity (over 25\% BW increase) and hyperglycemia (fasting glucose over $7 \mathrm{mmol} / \mathrm{L}$ ) were established, the mice were subjected to transient middle cerebral artery occlusion (tMCAO) (see Fig. 1a).

After tMCAO, HFD in the T2D group was replaced with a SD to reflect the clinical setting of a balanced poststroke diet. Mice fed SD from the beginning of the experiment will be referred to as Non-T2D while mice fed HFD until stroke time will be referred to as T2D.

One mouse from the Non-T2D group was removed due to unsuccessful tMCAO and 2 mice from the T2D group were removed shortly after surgery due to severe condition exceeding the allowed ethical break point. The remaining T2D mice were randomized in two groups 3 days after stroke: 1) one group was treated with daily intraperitoneal (i.p.) injections of $0.2 \mu \mathrm{g} / \mathrm{kg}$ Ex-4 (referred as T2D-Ex-4) for 8 weeks, 2) another group received vehicle (saline) injections (referred as T2D-Ve). Two mice from T2D-Ex-4 group were later removed since insufficient stroke damage was detected after histological examination. The final number of animals used in this study were as following: Non-T2D $(n=9), T 2 D-V e(n=9)$ and T2D-Ex$4(n=7)$. The forelimb sensorimotor function was then measured by assessing upper-limb grip strength for up-to 8 weeks after stroke when the mice were sacrificed (time when the non-T2D mice have fully recovered). Brains were then collected for histology. See Fig. 1a for the experimental design.

Study 2 (non-diabetic study): Twenty 8-months-old male C57BL/6J mice were used. Six mice were removed after surgery due to either unsuccessful tMCAO or severe condition. The remaining mice were randomized in two groups: 1$)$ Non-T2D-Ve $(n=6)$ and Non-T2D-Ex-4 $(n=8)$ and received daily vehicle or $0.2 \mu \mathrm{g} / \mathrm{kg}$ Ex-4 injections, respectively, starting from day 3 after tMCAO until 6 weeks. The forelimb sensorimotor function was tested as for Study 1 but this study was terminated 6 weeks after stroke (time when the non-T2D-Ex-4 mice have fully recovered). Brains were then collected for histology. See Fig. 1b for the experimental design. 


\section{Transient Middle Cerebral Artery Occlusion}

tMCAO was used to model ischemic stroke and was induced by the intraluminal filament technique as previously described (43). Briefly, mice were anesthetized by $3 \%$ isoflurane and then maintained by $1.5 \%$ isoflurane through a snout-mask throughout the surgery. Body temperature was maintained at $37-38^{\circ} \mathrm{C}$ using a heated pad. Through midline incision, left external (ECA) and internal (ICA) carotid arteries were exposed and a 7-0 monofilament coated with silicone (total diameter 0.17-0.18 mm) was inserted from an incision in ECA into the ICA until it blocked the origin of the MCA. Then the wounds were temporarily closed, and the mice were allowed to wake up. After 25 minutes, the mice were re-anesthetized, the wound re-opened and the occluding filament removed (total occlusion time $=30 \mathrm{~min}$ ). Stroke induction was considered unsuccessful when the occluding filament could not be advanced within the internal carotid artery beyond 7-8 $\mathrm{mm}$ from the carotid bifurcation or if mice lacked neurological impairment symptoms based on the neurological severity score (44).

\section{Fasting Glycaemia and Insulin tolerance test}

Fasting glycaemia measurement and insulin tolerance test (ITT) were performed before and at 4 and 8 weeks after stroke. Fasting glycaemia was measured after an overnight fasting. For ITT, mice were fasted for $6 \mathrm{~h}$ and injected i.p. with human insulin $(0.5 \mathrm{U} / \mathrm{kg})$ dissolved in saline. Blood glucose was determined before injection of insulin and at pre-set time points after injection (15, 30, 45, 60, 75 and $90 \mathrm{~min})$. Data are presented as area under the curve (AUC); see Fig. 2.

\section{Assessment of the recovery of forelimb sensorimotor function}

The forelimb sensorimotor function was measured by assessing upper-limb grip strength $(42,45)$ using a grip strength meter (Harvard apparatus, MA, USA) before, at 3 days and at 1-8 weeks after tMCAO. Briefly, mice were firmly held by the body and allowed to grasp the grid with the right forepaw. Mice were gently dragged backward until the grip was released. Ten trials were performed and the highest value was recorded as described previously $(42,45)$. The grip strength test was performed by an experimenter blinded to the treatment groups (Ex-4 vs. vehicle), but not diet (SD vs. HFD) due to the obvious weight differences during the first weeks after tMCAO.

\section{Immunohistochemistry and quantitative microscopy}

To prepare tissue for immunohistochemistry (IHC), the mice were anesthetized and transcardially perfused with $4 \%$ ice-cold paraformaldehyde before the brains were removed. After overnight post-fixation the brains were transferred to a solution of phosphate-buffered saline (PBS) with $20 \%$ sucrose until they sank. The brains were cut in $30-\mu \mathrm{m}$-thick coronal sections using a sliding microtome and kept in antifreeze solution at $-20^{\circ} \mathrm{C}$. 
Immunofluorescence staining was performed using the free-floating method. Briefly, brain sections were washed in PBS. For DAB stainings, sections were incubated with PBS containing $3 \% \mathrm{H}_{2} \mathrm{O}_{2}$ and $10 \%$ methanol for 20 min at RT to quench endogenous peroxidases. For both DAB and immunofluorescence stainings, sections were then blocked in PBS containing 3-5\% appropriate normal serum and 0,25\% Triton-X100 for $1 \mathrm{~h}$ (at RT), and incubated overnight in primary antibody solution (at RT for the CD31 and $\mathrm{CD} 13$ stainings and at $4^{\circ} \mathrm{C}$ for all other stainings). The following primary antibodies were used; mouse anti-NeuN (1:500 dilution; \#MAB377; Millipore), a neuronal marker; goat anti-lba-1 (1:1000 dilution; \#ab5076), a marker for microglia, and rabbit anti-CD68 (1:2000 dilution; \#ab125212), a marker for phagocytic microglia and macrophages; rabbit anti-parvalbumin (PV) (1:1,000 dilution; \#ab11427; Abcam), a marker of parvalbumin-expressing interneurons; rabbit anti-CD13 (1:200 dilution; \#MCA2183, Bio-RAD), a marker for pericytes; goat anti-CD31 (1:200 dilution; R\&D Systems \#AF3628), a marker for endothelial cells; rabbit anti-calretinin (CR) (1:400 dilution; \#ab92341), a marker for early post-mitotic neurons and calretinin-expressing interneurons; mouse-anti cFos (1:800 dilution, \#ab208942), a marker for neuronal activation; mouse anti-doublecortin (DCX) (1:200 dilution; \#sc-271390; Santa Cruz Biotechnology), a marker for migrating neuroblasts. After overnight incubation with the primary antibody solution, sections were washed and incubated for 1,5-2h at RT with the secondary antibody. For CD31, the signal was amplified by incubating sections with a biotinylated secondary antibody, followed by incubation with Alexa fluor-conjugated streptavidin.

The Olympus BX51 microscope coupled with computerized setup for stereology (Visiopharm, DK or MBF Biosciences, USA) was used for stroke volume measurement, lba-1 + cell counting and PV + cell volume measurements. Manual counting of CD68+, PV+, cFOS+, CR + and DCX + cells was performed on an Olympus BX40. For cell counting, three consecutive brain sections spaced at $300 \mu \mathrm{m}$ containing striatum (from Bregma 1 to $0.5 \mathrm{~mm}$ ) were used. The first section was chosen based on an anatomical location along the rostra-caudal axis (approximately $1 \mathrm{~mm}$ from Bregma). The second and the third sections were 300 and $600 \mu \mathrm{m}$ caudal from the first section, respectively. Microscopy was performed by experimenters blinded to experimental groups.

In addition to counting of Iba- 1 cells in the entire striatum, Iba- 1 cells were also counted in a specific region of interest (ROI) within the peri-infarct striatum. This ROI was defined as a $200 \mu \mathrm{m}$ wide zone, directly adjacent to the infarct region. The number of Iba- $1+$ cells within the ROI was counted (in both the ipsi- and contralateral striatum) and the cell density was expressed as number of cells $/ \mathrm{mm}^{2}$.

For analysis of CD31 + vessels and CD13 + pericytes, confocal images were sampled using a LEICA DMi8 confocal microscope. The images of the ipsilateral striatum overlapped both the infarct and peri-infarct region; on average, images covered $65 \%$ infarct region, and $35 \%$ peri-infarct region (determined based on NeuN staining). The collected images were obtained from a z-stack size of $10 \mu \mathrm{m}$ at a step size of $1 \mu \mathrm{m}$. For maximum image projection, Image J software (NIH, USA) was used for z-stack image reconstruction. The same acquisition settings were applied for each image. Blood vessel (CD31+) and pericyte (CD13+) density were analyzed using the area fraction measurement tool of Image J software ( $\mathrm{NIH}, \mathrm{USA})$. The density was expressed as the percentage of the CD31 + or CD13 + area. The coverage of vessels by CD13 
+ pericytes was assessed by calculating the proportion of CD13 + pericytes covering CD31 + vessels. We used the colocalization plugin tool of Image $(\mathrm{NIH}, \mathrm{USA})$ to define and highlight the covering points of CD13 + with CD31 + in the z-stack image. The images were reconstructed for a maximum projection and the $\mathrm{CD} 13+/ \mathrm{CD} 31+$ coverage area was measured and expressed as a percentage of the total CD31 + area per image. Parenchymal CD13 density was quantified separately by subtracting the density of the perivascular CD13 + cells from the total CD13 density.

\section{Stroke volume measurement}

Stroke volume was evaluated based on NeuN staining and a Cavalieri estimator probe (MBF Biosciences, USA) on all brain sections with visible stroke. NeuN staining is a consistent method for quantifying neural damage since it exclusively stains neurons. Therefore, it is reliable to evaluate neuronal loss even several weeks post-stroke unlike live cell markers like TTC (3,5-triphenyltetrazolium chloride) that accurately identify stroke-damaged tissue (by lack of positive stain) only within few days after the injury, due to later inflammatory cell infiltration and glial scar formation.

\section{Mouse insulin ELISA}

Serum insulin levels were determined using the Ultra-Sensitive Mouse Insulin ELISA Kit (CrystalChem, \#90080) according to manufacturer's instructions. A total sample volume of $10 \mu \mathrm{L}$ of serum was used per mouse.

\section{Statistical analysis}

All data were analyzed by GraphPad Prism Version 8. The data were first checked for statistical outliers, and normality using the Shapiro-Wilk normality test to decide whether to perform parametric or nonparametric tests. For the diabetic study we compared Non-T2D vs. T2D-Ve vs. T2D-Ex-4 mice. For the nondiabetic study Non-T2D-Ve vs. Non-T2D-Ex-4 mice were compared.

\section{Parametric tests}

Two-way repeated measures ANOVA was used to analyze grip strength in both the diabetic and nondiabetic study followed by Sidak's test. For body weight, fasting glucose and insulin tolerance test (ITT) in the diabetic study, two-way ANOVA followed by Tukey's or Bonferroni's test was performed. To analyze the volume and number of PV + cells, number of cFOS+/PV + and Iba- $1+$ cells, density of CD31 + vessels and CD13 + pericytes, and coverage of CD31 + vessels by CD13 + pericytes, two-way ANOVA followed by Two-stage linear step-up procedure of Benjamini, Krieger and Yekutieli test was performed. One-way ANOVA with Tukey's post-hoc test was used to analyze parenchymal CD13 density in the ipsilateral striatum, in the diabetic study. The number of DCX + cells in the ipsilateral striatum was analyzed using Brown-Forsythe and Welch ANOVA. In the non-diabetic study, the stroke volume and the number of CD68 + cells were analyzed using unpaired t-test.

\section{Non-parametric tests}


For the statistical analysis of stroke volume and CD68 + cells in the diabetic study, Kruskal-Wallis with uncorrected Dunn's test was performed.

All data are expressed as mean \pm SD. P-value less than 0.05 was considered statistically significant.

\section{Results}

Exendin-4 normalizes the T2D-induced impairment of neurological recovery after stroke in association with the normalization of glycemia and insulin resistance

To detect any potential effects of T2D and Ex-4 on stroke recovery in T2D mice and potentially correlate this effect with histological outcomes, the experiment was terminated at 8 weeks after TMCAO (time when the Non-T2D mice fully recovered forepaw grip strength after stroke).

There was no difference between the forepaw grip strength in Non-T2D and T2D mice before tMCAO (data not shown). At day 3 post-tMCAO, stroke decreased forepaw grip strength approximately by $40 \%$ (pre-tMCAO levels are indicated by the dashed line on Fig. 2a) in both Non-T2D and T2D mice (Fig. 2a). The two-way repeated measures ANOVA analysis revealed that during the recovery time (3 days -8 weeks), forepaw grip strength significantly increased in all mice (main effect - time, $p<0.0001$ ) (Fig. 2a). However, the grip strength was significantly greater in Non-T2D vs. T2D-Ve mice (main effect $-T 2 D, p<$ $0.0001)$ and a significant interaction $(p=0.0496)$ between time and T2D was recorded (Fig. 2a). These results show that T2D significantly worsened the grip strength recovery. The grip strength in T2D-Ex-4 mice was significantly greater vs. T2D-Ve mice (main effect - Ex-4 treatment, $p<0.0001$ ) and here too, a significant interaction between time and treatment ( $p=0.0008$, Fig. 2a) was recorded, indicating that Ex-4 significantly improved the grip strength in the recovery phase in T2D mice. Later, post-hoc statistical tests showed that Non-T2D mice fully recovered forepaw strength by 8 weeks reaching the pre-tMCAO levels (Fig. 2a), while in T2D-Ve mice the forepaw strength stayed significantly lower $(p<0.0001$, Fig. 2a) than in Non-T2D mice. Remarkably, T2D mice treated with Ex-4 showed a significant increase in grip strength $v s$. untreated T2D-Ve mice already from 3 weeks after stroke ( $p=0.0101$ Fig. $2 a)$ and onwards, and fully recovered their forepaw grip strength by week 8 . These differences in grip strength recovery were not determined by the differences in stroke severity, since no difference in ischemic volume was recorded between any of the groups (Fig. 2e-f).

T2D-Ve mice significantly and rapidly decreased body weight after TMCAO (and the substitution of HFD with SD) and from 1-2 weeks after stroke we could not observe any difference in body weight between the groups (Fig. 2b). As expected, fasted levels of blood glucose in T2D mice were significantly higher than in Non-T2D mice before tMCAO ( $p<0.0001$, Fig. $2 c)$. After tMCAO and the change from HFD to SD, blood glucose significantly decreased in the T2D-Ve group at both 4 (pre-tMCAO vs. 4 weeks post-tMCAO, $\mathrm{p}<0.0001)$ and 8 weeks ( 4 weeks post-tMCAO vs. 8 weeks post-tMCAO, $p<0.0001$ ) but still remained significantly higher $v s$. Non-T2D mice both at 4 weeks post-tMCAO $(p<0.0001)$ (Fig. $2 c)$ and at 8 weeks $(p=0.0043)$, although at this time-point the mean glucose levels in T2D-Ve mice decreased below the diabetic threshold of 7mmol/L (Fig. 2c). In T2D mice treated with Ex-4, fasted blood glucose of $\approx 6$ 
$\mathrm{mmol} / \mathrm{L}$ was reached already at 4 weeks after $\mathrm{tMCAO}$, although still significantly higher than the Non-T2D mice $(p<0.0001$, Fig. $2 \mathrm{c})$ and was completely normalized $(<5.6 \mathrm{mmol} / \mathrm{L})$ at 8 weeks after $\mathrm{tMCAO}$ (Fig. 2c). We also evaluated insulin sensitivity in all groups before stroke and at 4 and 8 weeks posttMCAO. Non-T2D mice maintained normal insulin sensitivity throughout the experiment and T2D-Ve mice remained insulin resistant (Fig. 2d). However, the T2D mice treated with Ex-4 showed improved insulin sensitivity already at 4 weeks post-tMCAO compared to T2D-Ve mice $(p<0.0001$, Fig. $2 \mathrm{~d})$, and then reached the levels of Non-T2D mice at 8 weeks after tMCAO (Fig. 2d).

In summary, these results show that despite normalization of body weight shortly (2 weeks) after tMCAO, during a large part of the post-stroke recovery phase (at least for 4 weeks) T2D-Ve mice remained hyperglycemic and for the entire recovery phase (8 weeks) they were insulin resistant. Ex-4 treatment in T2D mice normalized hyperglycemia already 4 weeks after tMCAO and gradually improved insulin sensitivity at 4 and 8 weeks post-tMCAO. We conclude that the poststroke decrease in body weight does not correlate with improvement of recovery in T2D. On the contrary, the impaired poststroke neurological recovery in T2D mice is associated with hyperglycemia and insulin resistance, and Ex-4 treatment improves stroke recovery by normalizing these parameters.

\section{Improved neurological recovery by Exendin-4 in T2D mice correlates with normalization of T2D-induced atrophy of GABAergic parvalbumin + interneurons, reduced inflammation and improved vascular remodeling and fibrotic scar formation}

Stroke similarly decreased $(\approx 60 \%)$ the number of surviving PV + interneurons in the ipsilateral striatum, in all experimental groups (Fig. 3a). We have previously shown that shortly (2 weeks) after stroke the soma volume of $\mathrm{PV}+$ interneurons is decreased in the peri-infarct region of the striatum and, while in NonT2D mice the soma volume recovers back to normal within 6 weeks after stroke, this atrophy persists in T2D (42). In the present study, potential differences in stroke-induced atrophy of PV + interneurons were also assessed by measuring the soma volume of these neurons in the contralateral and ipsilateral periinfarct striatum. The results in Fig. $3 \mathrm{~b}$ show a substantial atrophy of PV + interneuron soma volume in the ipsilateral peri-infarct striatum of T2D-Ve mice compared to the corresponding region in Non-T2D mice ( $p$ $=0.005)$ and compared to its own contralateral striatum $(p=0.0026)$, at 8 weeks after stroke. In Ex-4treated T2D mice, no differences were detected in the soma volume of PV + interneurons in the ipsilateralperi-infarct striatum vs Non-T2D mice or vs. its own contralateral striatum (Fig. 3b), indicating that the Ex4 treatment reversed PV + interneuron atrophy. To quantify potential differences in basal activation of PV + interneurons between the groups, we quantified the number of cFos/PV double-positive neurons in the peri-infarct striatum. A fraction of PV + interneurons was activated in both contra- and ipsilateral striatum, without differences between the groups (Additional file 1: Figure S1).

To assess potential changes in the neuroinflammatory response after stroke, we quantified lba- $1+$ and CD68 + microglia cells in the contralateral and ipsilateral striatum at 8 weeks post-tMCAO. The total density of Iba- $1+$ microglia in the ipsilateral versus respective contralateral striatum was significantly increased after stroke similarly in all groups, indicating persistent neuroinflammation at 8 weeks post- 
tMCAO without any additional effects of diabetes or Ex-4 treatment (Fig. 4a). However, when we evaluated density of Iba-1 + microglia specifically in infarct-adjacent areas (termed ROI, see Methods), we recorded a significant effect of Ex-4 in reducing Iba- $1+$ cell density in these regions $(p=0.0017$ for nonT2D vs. T2D-Ex-4, and a strong trend $p=0.0605$ for T2D-Ve vs T2D-Ex-4) (Fig. 4b). Microglia/macrophage activation (number of CD68 + cells) was also reduced by Ex-4 ( $p=0.0105$ non-T2D vs. T2D-Ex-4 and $p=$ 0.0595 T2D-Ve vs T2D-Ex-4) (Fig. 4c).

To assess any impact of T2D and Ex-4 treatment on vascular remodeling after stroke, CD31 + vessel density and maturity were examined. Striatal vessel density in the infarct area was increased in all groups compared to the respective contralateral striatum (contra vs ipsi: $p<0.0001$ for non-T2D, $p=0,0001$ for T2D-Ve and $p<0.0001$ for T2D-Ex-4) (Fig. 5b). T2D-Ve mice, however, had a significantly reduced ipsilateral striatal vessel density vs non-T2D mice $(p=0.0001)$, which was restored by Ex-4 treatment ( $p<$ 0.0001) (Fig. 5b). Notably, Ex-4 treatment increased vessel density in T2D mice to even slightly above non-T2D levels ( $p=0.0475$ ) (Fig. 5b). A similar pattern was found for the coverage of vessels by pericytes, an indicator of vessel maturity. In fact, the coverage of CD31 + vessels by CD13 + pericytes was increased by stroke in all groups, when comparing the ipsilateral vs contralateral striatum (contra vs ipsi: $p<0.0001$ for non-T2D, T2D-Ve and T2D-Ex-4) (Fig. 5C). However, pericyte coverage in the ipsilateral striatum was reduced in T2D mice when comparing T2D-Ve vs non-T2D mice $(p<0.0001)$ and normalized by Ex-4 treatment $(p<0.0001)$ (Fig. $5 c)$, indicating improved vascular remodeling and pericyte recruitment.

Similarly, the total pericyte density was increased after stroke in all groups when comparing the ipsilateral vs contralateral striatum (contra vs ipsi: $p<0.0001$ for non-T2D, $p=0.0076$ for T2D-Ve and $p<0.0001$ for T2D-Ex-4) (Fig. 5d). The total density of CD13 + pericytes in the ipsilateral striatum was reduced in T2DVe vs non-T2D mice $(p<0.0001)$ and increased by Ex-4 treatment $(p<0.0001$ T2D-Ve vs T2D-Ex-4) (Fig. 5d). Changes in CD13 + pericyte density between T2D-Ve vs non-T2D mice and the restorative effect of Ex-4 were observed for both perivascular and parenchymal pericytes, as indicated by the density of parenchymal pericytes in the ipsilateral striatum, an indicator of the fibrotic scar formation after stroke ( $p$ $=0.0061$ non-T2D vs T2D-Ve, $p=0.0301$ T2D-Ve vs T2D-Ex-4) (Fig. 5e). There were no differences between groups in the contralateral striatum for all analyses (Fig. $5 b-d$ ).

We also assessed potential changes in the early phase of stroke-induced neurogenesis by quantifying neuroblasts $(\mathrm{DCX}+)$ and early post-mitotic neurons (Calretinin+/NeuN-) (46) in the striatum 8 weeks after tMCAO. The number of DCX + cells was increased in ipsilateral $v s$. contralateral striatum in all groups, while no differences between ipsilateral $v s$. contralateral striatum were found for the number of Calretinin+/NeuN- cells (data not shown). We did not record any significant differences between the groups, for both of the assessed cell types (Additional file 1: Figure S3a,b).

Overall, these results suggest that T2D impairs recovery of forepaw grip strength during 8 weeks after stroke in association with a substantial atrophy of PV + interneurons and reduced vascular remodeling and fibrotic scar formation, and that this effect is counteracted by Ex-4 treatment. Ex-4 also reduced 
neuroinflammation, however we could not correlate this effect with improved neurological recovery since no differences in Iba-1 + and CD68 + microglia cells were observed between non-T2D and T2D-Ve mice.

\section{Exendin-4 marginally improved neurological recovery after stroke in non-T2D mice}

The next step in our study was to investigate the potential efficacy of Ex-4 to improve neurological recovery after stroke in non-T2D mice. In order to detect any potential effects of Ex-4 on stroke recovery in non-T2D mice and potentially correlate this effect with histological outcomes, the experiment was terminated at 6 weeks after IMCAO (time when the Ex-4-treated mice fully recovered forepaw grip strength after stroke). The two-way repeated measures ANOVA analyses showed a significant time effect $(\mathrm{p}<$ 0.0001) (both groups improved grip strength over time) and no significant treatment/Ex-4 effect (Fig. 6a). However, a significant interaction between time and Ex-4 treatment was still found ( $p=0.0069$, Fig. 6a), showing that the difference in grip strength between the groups was increasing over time. This indicates a positive effect of Ex-4 on neurological recovery that we interpret as minor in comparison with the effect obtained in the T2D study (Fig. 2a).

Histological/quantitative assessments of stroke volume (Fig. 6b), PV + interneuron volume (Fig. 6c), neuroinflammation (Fig. 6d, e), CD31 + vessel density (Fig. 6f), coverage of vessels by pericytes (Fig. $6 \mathrm{~g}$ ) and CD13 + pericyte density (Fig. 6h) did not reveal any significant effects of Ex-4 treatment. However, despite the mice being normoglycemic, we could detect a significant increase of plasma insulin levels in the Ex-4-treated group (Fig. 6i).

\section{Discussion}

We show that Ex-4 administered to diabetic mice after ischemic stroke leads to a significant improvement of post-stroke recovery assessed by forepaw grip strength (indicative of neurological functional recovery). This effect was associated with the normalization of hyperglycemia and insulin sensitivity, as well as with a reversal of stroke-induced PV + interneuron atrophy, and improvement of vascular remodeling as assessed by vascular density, coverage of vessels by pericytes and maintenance of fibrotic scar formation. Additionally, the Ex-4 treatment reduced stroke-induced inflammation. Under non-diabetic condition, Ex-4 treatment only slightly accelerated neurological recovery.

The medical need to identify new strategies to improve neurological recovery after stroke in the T2D population is urgent since the global prevalence of T2D is not only dramatically increasing but T2D is also one of the strongest risk factors for stroke (see Introduction). This will result in an exceptionally large increase of people with T2D in need of post-stroke treatment and care. Our results showing that Ex-4 improves post-stroke neurological recovery in T2D mice suggest that Ex-4 could be a valid candidate to meet this medical need. In fact, Ex-4 and other GLP-1R agonists could have several advantages. Indeed, the 2020 Standards of medical care for diabetes from the American Diabetes Association recommends the use of GLP-1R agonist with demonstrated cardiovascular benefit as an adjunct to metformin in T2D patients with high atherosclerotic cardiovascular disease (ASCVD) risk or established ASCVD for primary or secondary prevention of a cardiovascular event (47). A meta-analysis on the effect of GLP-1R agonists 
including pooled analysis of 56,004 patients encompassing the Lixisenatide (ELIXA), Liraglutide(LEADER), Semaglutide (SUSTAIN-6), Exenatide (synthetic form of Exendin-4) (EXSCEL), Albiglutide (HARMONY), Dulaglutide (REWIND) and Semaglutide (PIONEER-6) randomized control trials showed a $16 \%$ reduction in risk of total stroke, a $15 \%$ reduction in risk of nonfatal stroke, and a non-significant $19 \%$ reduction in fatal stroke (48). Furthermore, there is a vast literature indicating that GLP-1R activation can reduce acute brain damage after stroke in rodents with $(40,49)$ or without T2D (reviewed in $(13,50)$ ). Therefore, if our results will be confirmed in other experimental settings (and by using other GLP-1R agonists), they could reveal that a therapy based on GLP-1R activation in T2D patients, in addition to preventive and acute/reparative effects, could also present chronic beneficial effects on neurological recovery in T2D people suffering from stroke. Importantly, this question could also soon find an answer in the clinical setting. Indeed, the Short-Term Exenatide in Acute ischemic Stroke (STEXAS) study is currently testing the safety of Exenatide versus insulin in patients with hyperglycemia in the acute phase after ischemic stroke and will include the modified Rankin scale after 3 months in their secondary outcome analysis (48). In addition, the Treatment with Exenatide in Acute Ischemic Stroke (TEXAIS) phase 2 trial is currently including patients both with and without hyperglycemia in the acute phase after ischemic stroke to investigate neurological improvement at 7 days as a primary end-point and the modified Rankin scale after 90 days as a secondary outcome (51). However, of note, these studies will not evaluate the effect of Exenatide on a population with a previous T2D diagnosis and thus more studies will be needed in the future (14).

The mechanisms at the basis of improved neurological recovery mediated by Ex-4 are largely unknown. In our study we demonstrated that neurological recovery by Ex-4 did not correlate with weight loss during the recovery phase, as both vehicle and Ex-4-treated groups achieved normal weight similarly and simultaneously within the first weeks after tMCAO. However, Ex-4 treatment rapidly (already at 4 weeks) reduced fasting hyperglycemia and significantly improved insulin sensitivity and both these parameters were entirely normalized 8 weeks post-tMCAO by Ex-4. These results suggest the key importance of normalizing hyperglycemia to improve stroke recovery not only acutely after hospitalization, but also chronically for the entire poststroke recovery phase consistent with our previous reports where two other glycemic strategies (DPP-4 inhibitors and sulfonylurea) were employed (45).

Only a few clinical studies have so far investigated the potential detrimental role of insulin resistance on neurological recovery after stroke. A Chinese study of 1,245 non-diabetic patients with mild ischemic stroke observed a correlation between insulin resistance (measured by HOMA-IR) in the acute phase with worse functional outcome defined as modified Rankin score 3-6 after 1 year with an adjusted odds ratio 1.42 (1.03-1.95) (52). Moreover, Ozkul and colleagues examined the relationships between inflammation, oxidative stress and stroke severity in a small study of 75 acute stroke patients with and without insulin resistance. They found increased IL- 6 and decreased IL-10 levels in insulin-resistant patients and a correlation with higher NIHSS scores indicating more severe stroke (53). Finally, a Japanese registry study by Ago et al analyzed 4,655 patients with ischemic stroke correlated insulin resistance (measured by HOMA-IR) with worse functional outcome in non-insulin dependent patients (54). Although speculative, 
the results of these clinical studies support the potential role of Ex-4 to improve neurological recovery after stroke through the normalization of insulin resistance.

The positive effects mediated by Ex-4 in stroke recovery could also be mediated by indirect (via glycemia regulation and normalization of insulin resistance) or direct effects occurring in the brain. Interestingly, we show that Exe-4 treatment reversed T2D-induced PV + interneuron atrophy (42). PV + interneurons play key role in neuronal plasticity and thus could have significant effects on neurological recovery after stroke $(55,56)$. It is difficult to determine whether the efficacy of Ex-4 on reversing PV + interneuron atrophy could be linked to metabolic (reduced fasting hyperglycemia and improved insulin sensitivity) vs. direct neuroplasticity effects. Analyzing Ex-4 effects on PV + interneuron atrophy in non-diabetic conditions should have answered this question. However, in the non-diabetic study, PV + interneuron volume was already normalized in vehicle group at 6 weeks after stroke when the morphometric analyses were performed. Thus, whether Ex-4 had any effects on PV + interneuron volume normalization at earlier time-points remains to be investigated.

Ex-4 treatment reduced stroke-induced inflammation in T2D, but not in non-T2D mice. Although the antiinflammatory efficacy of Ex-4 after stroke is well-established in preclinical studies $(31,57)$, in the present study we could not correlate this effect with improvement in stroke recovery since we could not detect any increase of neuroinflammation in T2D vs. non-T2D mice at 8 weeks after stroke. However, considering the vast literature regarding anti-inflammatory efficacy of GLP-1 analogues (58), the potential effects of Ex-4 treatment at earlier time-points should not be discounted.

Poststroke recovery during the chronic phase requires remodeling of the vasculature (59). Remodeling of the vasculature is closely associated with vessel stabilization by brain pericytes (60-63). Impaired poststroke vessel density in T2D indicates that diabetic conditions reduce recovery mechanisms under pathological situations, such as stroke (64). Consistently with previous reports, we found that vessel density and coverage of vessels by pericytes were significantly reduced in T2D mice after stroke indicating failure to form new mature microvessels $(65,66)$. Interestingly, we show for the first time that this negative impact of T2D on vascular remodeling in the chronic phase of stroke could be completely reversed by Ex-4 treatment. This novel finding is in line with previously reported beneficial effects of GLP$1 \mathrm{R}$ activation on the vasculature. For example, GLP-1 has been shown to improve endothelial function in T2D (27) and GLP-1R activation after stroke can stimulate angiogenesis $(29,30)$, microvascular recruitment and blood flow (67).

Pericytes play an active role in tissue remodeling after stroke. In particular, it has been suggested that pericytes detach from the vessel wall and migrate into the injured parenchyma where parenchymal pericytes form a dense network contributing to the fibrotic scar after stroke (69-72). Interestingly, our study shows that the density of parenchymal pericytes is reduced in T2D but is restored to comparable levels of non-diabetic animals when Ex-4 is administered. Even though the fibrotic scar is still scarcely studied in stroke, our results suggest that Ex-4 may play a beneficial role in tissue remodeling during the chronic phase of stroke. 
In the non-diabetic study, we showed that Ex-4 was only marginally effective to improve neurological recovery after stroke. This was surprising considering the vast literature showing the beneficial effects mediated by GLP-1R activation in rodent stroke models without T2D (12). Decreased efficacy under nondiabetic conditions could be explained by the already very robust recovery in vehicle-treated non-T2D mice (a ceiling effect). Indeed, these mice recover quickly after stroke thus likely providing only small opportunity to further improve recovery after pharmacological treatment. Another important aspect to consider is that in addition to the normalization of hyperglycemia and insulin sensitivity, GLP-1 analogues (and by proxy insulin) also improve/enhance synaptic- and neuroplasticity $(35,37,73,74)$. Furthermore several studies have shown the neurotrophic and neuroprotective effects of insulin and insulin-enhancing drugs in animal models of stroke $(75,76)$. Thus, the likelihood of the beneficial effects mediated by insulin on stroke recovery should not be discounted. We show that despite SD-fed mice (both untreated and treated with Ex-4) were normoglycemic, Ex-4 led to a significant increase of plasma insulin levels 6 weeks after IMCAO. Therefore, although speculative, the minor but significant effect of Ex-4 to improve stroke recovery could be potentially attributed to increased trophic/neuroplasticity mechanisms induced by elevated insulin.

Obvious limitations of the study are the lack of histological assessments at intermediate time-points and the reliance on a well-validated $(26,42,77,78)$, but single behavioral readout. Therefore, we acknowledge that our findings will have to be confirmed by additional studies. On the other hand, a strength of our study is represented by the employed experimental T2D model and clinically relevant study design. Unlike genetic or toxin-induced T2D models, diet-induced obesity/T2D more accurately models lifestyle-induced T2D in humans (79). Moreover, this model perfectly replicates the T2D-induced impairment of functional recovery that is observed in stroke patients (21). Additionally, by replacing HFD with SD after stroke we have removed the potential confounding effects of different diets during the stroke recovery phase, as well as mimicked the condition of diabetic stroke survivors who experiences lifestyle/diet changes after stroke.

\section{Conclusion}

In conclusion, our results demonstrate the efficacy of GLP-1R activation to improve neurological recovery after stroke in T2D. Our study expands on a previous report showing the significant benefit of glycemia regulation during the post-acute, chronic, stroke recovery phase (26) and providing the first experimental evidence for the key role of the normalization of insulin sensitivity to improve long-term neurological recovery after stroke. These results strongly motivate additional studies to investigate the use of T2D therapies that in addition to glycemia regulation also improve insulin sensitivity thus promoting better functional recovery after stroke.

\section{Abbreviations}

ASCVD: Atherosclerotic cardiovascular disease 

AUC: $\quad$ Area under the curve
BBB: $\quad$ Blood brain barrier
BW: $\quad$ Body weight
CD13: $\quad$ Cluster of differentiation 13/Aminopeptidase N
CD31: Cluster of differentiation 31/Platelet endothelial cell adhesion molecule
CD68: $\quad$ Cluster of differentiation 68
CR: $\quad$ Calretinin
DCX: $\quad$ Doublecortin
DPP-4i: $\quad$ Dipeptidyl peptidase-4 inhibitor
ECA: $\quad$ External carotid artery
GABA: $\quad$ Gamma aminobutyric acid
GLP-1: $\quad$ Glucagon-like peptide 1
GLP-1R: Glucagon-like peptide 1 receptor
HFD: $\quad$ High-fat diet
lba-1: $\quad$ lonized calcium-binding adapter molecule 1
ICA: Internal carotid artery
IHC: $\quad$ Immunohistochemistry
IL-6: $\quad$ Interleukin-6
IL-10: Interleukin-10
ITT: Insulin tolerance test
MCA: $\quad$ Middle cerebral artery
NeuN: $\quad$ Neuronal nuclei
NIHSS: National Institute of Health Stroke Scale
NSC: $\quad$ Neural stem cell 
PBS: $\quad$ Phosphate-buffered saline

ROI: $\quad$ Region of interest

SD: $\quad$ Standard laboratory diet

SVZ: $\quad$ Subventricular zone

T2D: $\quad$ Type 2 diabetes

tMCAO: Transient middle cerebral artery occlusion

TTC: $\quad$ 3,5-triphenyltetrazolium chloride

\section{Declarations}

\section{AVAILABILITY OF DATA AND MATERIALS}

The datasets used and/or analyzed during the current study are available from the corresponding author on reasonable request.

\section{ACKNOWLEDGEMENTS}

We thank Valentina Gustafsson for help with the IHC analysis, Dr. Fuad Bahram (Södersjukhuset) for technical assistance and Dr. Hans Pettersson for advice on statistical analyses.

\section{FUNDING}

Financial support was provided by the Swedish Research Council (Vetenskapsrådet), the European Foundation for the Study of Diabetes (EFSD)/Sanofi European Diabetes Research Program in Macrovascular Complications, the Swedish Heart-Lung Foundation, Diabetesfonden, Ulla Hamberg Angeby och Lennart Angebys Stiftelse, Svensk Förening för Diabetologi, Karolinska Institutet (Foundation for Geriatric Diseases and KI Stiftelser och Fonder), O. E. och Edla Johanssons Stiftelse, Magnus Bergvalls Stiftelse, STROKE Riksförbundet and by the regional agreement on medical training and clinical research (ALF) between Stockholm County Council and the Karolinska Institutet.

\section{AUTHOR INFORMATION}

\section{Affiliations}

The NeuroCardioMetabol Group, Department of Clinical Science and Education, Södersjukhuset, Internal Medicine, Karolinska Institutet, 11883 Stockholm, Sweden.

Ingrid Lovise Augestad, Doortje Dekens, Dimitra Karampatsi, Alexander Zabala, Hiranya Pintana, Martin Larsson, Thomas Nyström, Vladimer Darsalia and Cesare Patrone. 
Translational Neurology Group, Department of Clinical Sciences, Wallenberg Neuroscience Center, Wallenberg Center for Molecular Medicine, Lund University, 22184 Lund, Sweden.

Osama Elabi and Gesine Paul.

\section{Contributions}

I.A. and D.D performed behavioral analysis, immunohistochemistry studies and stereology analysis; acquired and processed images and figures; contributed to discussion; edited the manuscript. D.K. and O.E. acquired and processed images and figures, performed part of the immunohistochemistry, the ELISA experiments, contributed to discussion, and edited the manuscript. H.P. performed the stroke volume assessment. A.Z and M.L. provided expertise, contributed to discussion, and edited the manuscript T.N. provided expertise and resources, contributed to discussion and edited the manuscript. G.P. conceived and provided resources for the vascular part of the study, contributed to discussion, and edited the manuscript. V.D. conceived and designed the study, performed the stroke experiments and diabetic tests, contributed to discussion, and wrote the manuscript. C.P. conceived, designed, and coordinated the research plan, contributed to discussion, and wrote the manuscript. All authors read and approved the final manuscript.

\section{Corresponding authors}

Correspondence to Vladimer Darsalia and Cesare Patrone.

\section{ETHICS DECLARATIONS}

\section{Ethics approval and consent to participate}

All applicable international, national, and/or institutional guidelines for the care and use of animals were followed where the studies were conducted under the ethical approval ID1126 (Karolinska Institutet).

\section{Consent for publication}

Not applicable. All authors have declared their consent for this publication.

\section{Competing interests}

T.N. has received unrestricted grants from AstraZeneca and consultancy fees from Boehringer Ingelheim, Eli Lilly, Novo Nordisk, Merck and Sanofi-Aventis. The other authors declare that they have no competing interests.

\section{References}

1. Holz Iv GG, Kiihtreiber WM, Habener JF. Pancreatic beta-cells are rendered glucose-competent by the insulinotropic hormone glucagon-like peptide-1(7-37). Nature. 1993;361(6410):362-5. 
2. Gutniak M, Ørkov C, Holst JJ, Ahrén B, Efendić S. Antidiabetogenic Effect of Glucagon-like Peptide-1 (7-36)amide in Normal Subjects and Patients with Diabetes Mellitus. N Engl J Med. 1992;326(20):1316-22.

3. Gutniak MK, Linde B, Holst JJ, Efendic S. Subcutaneous injection of the incretin hormone glucagonlike peptide 1 abolishes postprandial glycemia in NIDDM. Diabetes Care. 1994;17(9):1039-44.

4. Zimmerman RS, Hobbs TM, Wells BJ, Kong SX, Kattan MW, Bouchard J, et al. Association of glucagon-like peptide-1 receptor agonist use and rates of acute myocardial infarction, stroke and overall mortality in patients with type 2 diabetes mellitus in a large integrated health system. Diabetes Obes Metab. 2017;19(11):1555-61.

5. Best JH, Hoogwerf BJ, Herman WH, Pelletier EM, Smith DB, Wenten M, et al. Risk of cardiovascular disease events in patients with type 2 diabetes prescribed the glucagon-like peptide 1 (GLP-1) receptor agonist exenatide twice daily or other glucose-lowering therapies: a retrospective analysis of the LifeLink database. Diabetes Care. 2011;34(1):90-5.

6. Paul SK, Klein K, Maggs D, Best JH. The association of the treatment with glucagon-like peptide-1 receptor agonist exenatide or insulin with cardiovascular outcomes in patients with type 2 diabetes: a retrospective observational study. Cardiovasc Diabetol. 2015;14:10.

7. Marso SP, Bain SC, Consoli A, Eliaschewitz FG, Jodar E, Leiter LA, et al. Semaglutide and Cardiovascular Outcomes in Patients with Type 2 Diabetes. N Engl J Med. 2016;375(19):1834-44.

8. Marso SP, Daniels GH, Brown-Frandsen K, Kristensen P, Mann JF, Nauck MA, et al. Liraglutide and Cardiovascular Outcomes in Type 2 Diabetes. N Engl J Med. 2016;375(4):311-22.

9. Hernandez AF, Green JB, Janmohamed S, D'Agostino RB, Sr., Granger CB, Jones NP, et al. Albiglutide and cardiovascular outcomes in patients with type 2 diabetes and cardiovascular disease (Harmony Outcomes): a double-blind, randomised placebo-controlled trial. Lancet. 2018;392(10157):1519-29.

10. Gerstein HC, Hart R, Colhoun HM, Diaz R, Lakshmanan M, Botros FT, et al. The effect of dulaglutide on stroke: an exploratory analysis of the REWIND trial. The lancet Diabetes endocrinology. 2020;8(2):106-14.

11. Alfayez OM, Almohammed OA, Alkhezi OS, Almutairi AR, Al Yami MS. Indirect comparison of glucagon like peptide-1 receptor agonists regarding cardiovascular safety and mortality in patients with type 2 diabetes mellitus: network meta-analysis. Cardiovasc Diabetol. 2020;19(1):96.

12. Marlet IR, Olmestig JNE, Vilsboll T, Rungby J, Kruuse C. Neuroprotective Mechanisms of Glucagonlike Peptide-1-based Therapies in Ischaemic Stroke: A Systematic Review based on Pre-Clinical Studies. Basic Clin Pharmacol Toxicol. 2018.

13. Darsalia V, Klein T, Nystrom T, Patrone C. Glucagon-like receptor 1 agonists and DPP-4 inhibitors: Anti-diabetic drugs with anti-stroke potential. Neuropharmacology. 2017.

14. Darsalia V, Larsson M, Klein T, Patrone $C$. The high need for trials assessing functional outcome after stroke rather than stroke prevention with GLP-1 agonists and DPP-4 inhibitors. Cardiovasc Diabetol. $2018 ; 17(1): 32$. 
15. Benjamin EJ, Virani SS, Callaway CW, Chamberlain AM, Chang AR, Cheng S, et al. Heart Disease and Stroke Statistics-2018 Update: A Report From the American Heart Association. Circulation. 2018;137(12):e67-492.

16. Zabala A, Darsalia V, Holzmann MJ, Franzen S, Svensson AM, Eliasson B, et al. Risk of first stroke in people with type 2 diabetes and its relation to glycaemic control: A nationwide observational study. Diabetes Obes Metab. 2019.

17. Rawshani A, Rawshani A, Franzen S, Sattar N, Eliasson B, Svensson AM, et al. Risk Factors, Mortality, and Cardiovascular Outcomes in Patients with Type 2 Diabetes. N Engl J Med. 2018;379(7):633-44.

18. Shaw JE, Sicree RA, Zimmet PZ. Global estimates of the prevalence of diabetes for 2010 and 2030. Diabetes Res Clin Pract. 2010;87(1):4-14.

19. Pulsinelli WA, Levy DE, Sigsbee B, Scherer P, Plum F. Increased damage after ischemic stroke in patients with hyperglycemia with or without established diabetes mellitus. Am J Med. 1983;74(4):540-4.

20. Megherbi SE, Milan C, Minier D, Couvreur G, Osseby GV, Tilling K, et al. Association between diabetes and stroke subtype on survival and functional outcome 3 months after stroke: data from the European BIOMED Stroke Project. Stroke. 2003;34(3):688-94.

21. Ullberg T, Zia E, Petersson J, Norrving B. Changes in functional outcome over the first year after stroke: an observational study from the Swedish stroke register. Stroke. 2015;46(2):389-94.

22. Luitse MJ, Biessels GJ, Rutten GE, Kappelle LJ. Diabetes, hyperglycaemia, and acute ischaemic stroke. Lancet Neurol. 2012;11(3):261-71.

23. Luitse MJ, van Seeters T, Horsch AD, Kool HA, Velthuis BK, Kappelle LJ, et al. Admission hyperglycaemia and cerebral perfusion deficits in acute ischaemic stroke. Cerebrovasc Dis. 2013;35(2):163-7.

24. Baird TA, Parsons MW, Phan T, Butcher KS, Desmond PM, Tress BM, et al. Persistent poststroke hyperglycemia is independently associated with infarct expansion and worse clinical outcome. Stroke. 2003;34(9):2208-14.

25. Palaiodimou L, Lioutas VA, Lambadiari V, Paraskevas GP, Voumvourakis K, Tsivgoulis G. Glycemia management in acute ischemic stroke: current concepts and novel therapeutic targets. Postgrad Med. 2019;131(7):423-37.

26. Augestad IL, Pintana H, Larsson M, Krizhanovskii C, Nystrom T, Klein T, et al. The Regulation of Glycemia in the Recovery Phase After Stroke Counteracts the Detrimental Effect of Obesity-Induced Type 2 Diabetes on Neurological Recovery. Diabetes. 2020.

27. Nystrom T, Gutniak MK, Zhang Q, Zhang F, Holst JJ, Ahren B, et al. Effects of glucagon-like peptide-1 on endothelial function in type 2 diabetes patients with stable coronary artery disease. American journal of physiology Endocrinology metabolism. 2004;287(6):E1209-15.

28. Tuttolomondo A, Cirrincione A, Casuccio A, Del Cuore A, Daidone M, Di Chiara T, et al. Efficacy of dulaglutide on vascular health indexes in subjects with type 2 diabetes: a randomized trial. Cardiovasc Diabetol. 2021;20(1):1. 
29. Chen Y, Zhang X, He J, Xie Y, Yang Y. Delayed Administration of the Glucagon-Like Peptide 1 Analog Liraglutide Promoting Angiogenesis after Focal Cerebral Ischemia in Mice. J Stroke Cerebrovasc Dis. 2018;27(5):1318-25.

30. Zhao L, Xu J, Wang Q, Qian Z, Feng W, Yin X, et al. Protective effect of rhGLP-1 (7-36) on brain ischemia/reperfusion damage in diabetic rats. Brain Res. 2015;1602:153-9.

31. Kim S, Jeong J, Jung HS, Kim B, Kim YE, Lim DS, et al. Anti-inflammatory Effect of Glucagon Like Peptide-1 Receptor Agonist, Exendin-4, through Modulation of IB1/JIP1 Expression and JNK Signaling in Stroke. Experimental neurobiology. 2017;26(4):227-39.

32. Shan $Y$, Tan $S$, Lin $Y$, Liao S, Zhang B, Chen $X$, et al. The glucagon-like peptide-1 receptor agonist reduces inflammation and blood-brain barrier breakdown in an astrocyte-dependent manner in experimental stroke. J Neuroinflamm. 2019;16(1):242.

33. Chen F, Wang W, Ding H, Yang Q, Dong Q, Cui M. The glucagon-like peptide-1 receptor agonist exendin-4 ameliorates warfarin-associated hemorrhagic transformation after cerebral ischemia. $J$ Neuroinflamm. 2016;13(1):204.

34. Johnstone A, Levenstein JM, Hinson EL, Stagg CJ. Neurochemical changes underpinning the development of adjunct therapies in recovery after stroke: A role for GABA? J Cereb Blood Flow Metab. 2018;38(9):1564-83.

35. Korol SV, Jin Z, Babateen O, Birnir B. GLP-1 and exendin-4 transiently enhance GABAA receptormediated synaptic and tonic currents in rat hippocampal CA3 pyramidal neurons. Diabetes. 2015;64(1):79-89.

36. Lietzau G, Nystrom T, Ostenson CG, Darsalia V, Patrone C. Type 2 diabetes-induced neuronal pathology in the piriform cortex of the rat is reversed by the GLP-1 receptor agonist Exendin-4. Oncotarget. 2016.

37. Larsson M, Lietzau G, Nathanson D, Ostenson CG, Mallard C, Johansson ME, et al. Diabetes negatively affects cortical and striatal GABAergic neurons; an effect that is partially counteracted by Exendin-4. Bioscience reports. 2016.

38. Dillen Y, Kemps H, Gervois P, Wolfs E, Bronckaers A. Adult Neurogenesis in the Subventricular Zone and Its Regulation After Ischemic Stroke: Implications for Therapeutic Approaches. Translational stroke research. 2020;11(1):60-79.

39. Yang X, Feng P, Zhang X, Li D, Wang R, Ji C, et al. The diabetes drug semaglutide reduces infarct size, inflammation, and apoptosis, and normalizes neurogenesis in a rat model of stroke. Neuropharmacology. 2019;158:107748.

40. Darsalia V, Mansouri S, Ortsater H, Olverling A, Nozadze N, Kappe C, et al. Glucagon-like peptide-1 receptor activation reduces ischemic brain damage following stroke in type 2 diabetic rats. Clin Sci (Lond). 2011.

41. Goke R, Fehmann HC, Linn T, Schmidt $H$, Krause M, Eng J, et al. Exendin-4 is a high potency agonist and truncated exendin-(9-39)-amide an antagonist at the glucagon-like peptide 1-(7-36)-amide receptor of insulin-secreting beta-cells. J Biol Chem. 1993;268(26):19650-5. 
42. Pintana H, Lietzau G, Augestad IL, Chiazza F, Nystrom T, Patrone C, et al. Obesity-induced type 2 diabetes impairs neurological recovery after stroke in correlation with decreased neurogenesis and persistent atrophy of parvalbumin-positive interneurons. Clin Sci (Lond). 2019;133(13):1367-86.

43. Hara H, Huang PL, Panahian N, Fishman MC, Moskowitz MA. Reduced brain edema and infarction volume in mice lacking the neuronal isoform of nitric oxide synthase after transient MCA occlusion. J Cereb Blood Flow Metab. 1996;16(4):605-11.

44. Bederson JB, Pitts LH, Tsuji M, Nishimura MC, Davis RL, Bartkowski H. Rat middle cerebral artery occlusion: evaluation of the model and development of a neurologic examination. Stroke. 1986;17(3):472-6.

45. Augestad IL, Pintana H, Larsson M, Krizhanovskii C, Nystrom T, Klein T, et al. Regulation of Glycemia in the Recovery Phase After Stroke Counteracts the Detrimental Effect of Obesity-Induced Type 2 Diabetes on Neurological Recovery. Diabetes. 2020;69(9):1961-73.

46. Brandt MD, Jessberger S, Steiner B, Kronenberg G, Reuter K, Bick-Sander A, et al. Transient calretinin expression defines early postmitotic step of neuronal differentiation in adult hippocampal neurogenesis of mice. Mol Cell Neurosci. 2003;24(3):603-13.

47. American Diabetes A. Standards of Medical Care in Diabetes-2020 Abridged for Primary Care Providers. Clin Diabetes. 2020;38(1):10-38.

48. Bellastella G, Maiorino MI, Longo M, Scappaticcio L, Chiodini P, Esposito K, et al. Glucagon-Like Peptide-1 Receptor Agonists and Prevention of Stroke Systematic Review of Cardiovascular Outcome Trials With Meta-Analysis. Stroke. 2020;51(2):666-9.

49. Darsalia V, Hua S, Larsson M, Mallard C, Nathanson D, Nystrom T, et al. Exendin-4 reduces ischemic brain injury in normal and aged type 2 diabetic mice and promotes microglial $\mathrm{m} 2$ polarization. PLoS One. 2014;9(8):e103114.

50. Maskery MP, Holscher C, Jones SP, Price Cl, Strain WD, Watkins CL, et al. Glucagon like peptide-1 receptor agonists as neuroprotective agents for ischemic stroke: a systematic scoping review. $\mathrm{J}$ Cereb Blood Flow Metab. 2020:271678X20952011.

51. Muller C, Cheung NW, Dewey H, Churilov L, Middleton S, Thijs V, et al. Treatment with exenatide in acute ischemic stroke trial protocol: A prospective, randomized, open label, blinded end-point study of exenatide vs. standard care in post stroke hyperglycemia. Int J Stroke. 2018;13(8):857-62.

52. Jing J, Pan Y, Zhao X, Zheng H, Jia Q, Mi D, et al. Insulin Resistance and Prognosis of Nondiabetic Patients With Ischemic Stroke: The ACROSS-China Study (Abnormal Glucose Regulation in Patients With Acute Stroke Across China). Stroke. 2017;48(4):887-93.

53. Ozkul A, Ayhan M, Akyol A, Turgut ET, Kadikoylu G, Yenisey C. The effect of insulin resistance on inflammatory response and oxidative stress in acute cerebral ischemia. Neuro Endocrinol Lett. 2013;34(1):52-7.

54. Ago T, Matsuo R, Hata J, Wakisaka Y, Kuroda J, Kitazono T, et al. Insulin resistance and clinical outcomes after acute ischemic stroke. Neurology. 2018;90(17):e1470-e7. 
55. Hattori R, Kuchibhotla KV, Froemke RC, Komiyama T. Functions and dysfunctions of neocortical inhibitory neuron subtypes. Nat Neurosci. 2017;20(9):1199-208.

56. Hu H, Gan J, Jonas P. Interneurons. Fast-spiking, parvalbumin(+) GABAergic interneurons: from cellular design to microcircuit function. Science. 2014;345(6196):1255263.

57. Darsalia V, Mansouri S, Ortsater H, Olverling A, Nozadze N, Kappe C, et al. Glucagon-like peptide-1 receptor activation reduces ischaemic brain damage following stroke in Type 2 diabetic rats. Clin Sci (Lond). 2012;122(10):473-83.

58. Marlet IR, Olmestig JNE, Vilsboll T, Rungby J, Kruuse C. Neuroprotective Mechanisms of Glucagonlike Peptide-1-based Therapies in Ischaemic Stroke: A Systematic Review based on Pre-Clinical Studies. Basic Clin Pharmacol Toxicol. 2018;122(6):559-69.

59. Liu J, Wang Y, Akamatsu Y, Lee CC, Stetler RA, Lawton MT, et al. Vascular remodeling after ischemic stroke: mechanisms and therapeutic potentials. Prog Neurobiol. 2014;115:138-56.

60. Kokovay E, Li L, Cunningham LA. Angiogenic recruitment of pericytes from bone marrow after stroke. J Cereb Blood Flow Metab. 2006;26(4):545-55.

61. Zechariah A, EIAli A, Doeppner TR, Jin F, Hasan MR, Helfrich I, et al. Vascular endothelial growth factor promotes pericyte coverage of brain capillaries, improves cerebral blood flow during subsequent focal cerebral ischemia, and preserves the metabolic penumbra. Stroke. 2013;44(6):1690-7.

62. Jean LeBlanc N, Guruswamy R, ElAli A. Vascular Endothelial Growth Factor Isoform-B Stimulates Neurovascular Repair After Ischemic Stroke by Promoting the Function of Pericytes via Vascular Endothelial Growth Factor Receptor-1. Mol Neurobiol. 2018;55(5):3611-26.

63. Ghori A, Freimann FB, Nieminen-Kelhä M, Kremenetskaia I, Gertz K, Endres M, et al. EphrinB2 Activation Enhances Vascular Repair Mechanisms and Reduces Brain Swelling After Mild Cerebral Ischemia. Arterioscler Thromb Vasc Biol. 2017;37(5):867-78.

64. Ergul A, Abdelsaid M, Fouda AY, Fagan SC. Cerebral neovascularization in diabetes: implications for stroke recovery and beyond. J Cereb Blood Flow Metab. 2014;34(4):553-63.

65. Prakash R, Li W, Qu Z, Johnson MA, Fagan SC, Ergul A. Vascularization pattern after ischemic stroke is different in control versus diabetic rats: relevance to stroke recovery. Stroke. 2013;44(10):2875-82.

66. Cui X, Chopp M, Zacharek A, Ye X, Roberts C, Chen J. Angiopoietin/Tie2 pathway mediates type 2 diabetes induced vascular damage after cerebral stroke. Neurobiol Dis. 2011;43(1):285-92.

67. Almutairi M, Al Batran R, Ussher JR. Glucagon-like peptide-1 receptor action in the vasculature. Peptides. 2019;111:26-32.

68. Lin WJ, Ma XF, Hao M, Zhou HR, Yu XY, Shao N, et al. Liraglutide attenuates the migration of retinal pericytes induced by advanced glycation end products. Peptides. 2018;105:7-13.

69. Fernández-Klett F, Potas JR, Hilpert D, Blazej K, Radke J, Huck J, et al. Early loss of pericytes and perivascular stromal cell-induced scar formation after stroke. J Cereb Blood Flow Metab. 2013;33(3):428-39. 
70. Makihara N, Arimura K, Ago T, Tachibana M, Nishimura A, Nakamura K, et al. Involvement of plateletderived growth factor receptor $\beta$ in fibrosis through extracellular matrix protein production after ischemic stroke. Exp Neurol. 2015;264:127-34.

71. Roth M, Gaceb A, Enström A, Padel T, Genové G, Özen I, et al. Regulator of G-protein signaling 5 regulates the shift from perivascular to parenchymal pericytes in the chronic phase after stroke. Faseb j. 2019;33(8):8990-8.

72. Shen J, Ishii Y, Xu G, Dang TC, Hamashima T, Matsushima T, et al. PDGFR- $\beta$ as a positive regulator of tissue repair in a mouse model of focal cerebral ischemia. J Cereb Blood Flow Metab. 2012;32(2):353-67.

73. McClean PL, Gault VA, Harriott P, Holscher C. Glucagon-like peptide-1 analogues enhance synaptic plasticity in the brain: a link between diabetes and Alzheimer's disease. Eur J Pharmacol. 2010;630(1-3):158-62.

74. Mainardi M, Fusco S, Grassi C. Modulation of hippocampal neural plasticity by glucose-related signaling. Neural plasticity. 2015;2015:657928.

75. Pomytkin I, Costa-Nunes JP, Kasatkin V, Veniaminova E, Demchenko A, Lyundup A, et al. Insulin receptor in the brain: Mechanisms of activation and the role in the CNS pathology and treatment. CNS Neurosci Ther. 2018;24(9):763-74.

76. Mielke JG, Wang YT. Insulin, synaptic function, and opportunities for neuroprotection. Prog Mol Biol Transl Sci. 2011;98:133-86.

77. Chiazza F, Pintana H, Lietzau G, Nystrom T, Patrone C, Darsalia V. The Stroke-Induced Increase of Somatostatin-Expressing Neurons is Inhibited by Diabetes: A Potential Mechanism at the Basis of Impaired Stroke Recovery. Cell Mol Neurobiol. 2020.

78. Chiazza F, Tammen H, Pintana H, Lietzau G, Collino M, Nystrom T, et al. The effect of DPP-4 inhibition to improve functional outcome after stroke is mediated by the SDF-1alpha/CXCR4 pathway. Cardiovasc Diabetol. 2018;17(1):60.

79. Heydemann A. An Overview of Murine High Fat Diet as a Model for Type 2 Diabetes Mellitus. J Diabetes Res. 2016;2016:2902351.

\section{Figures}




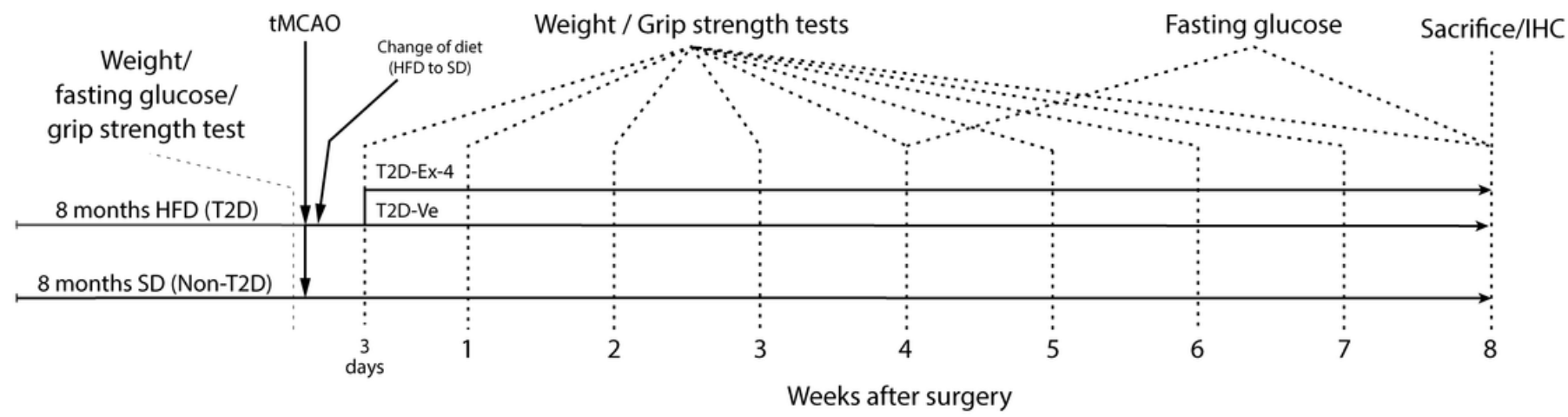

b Non-diabetic study

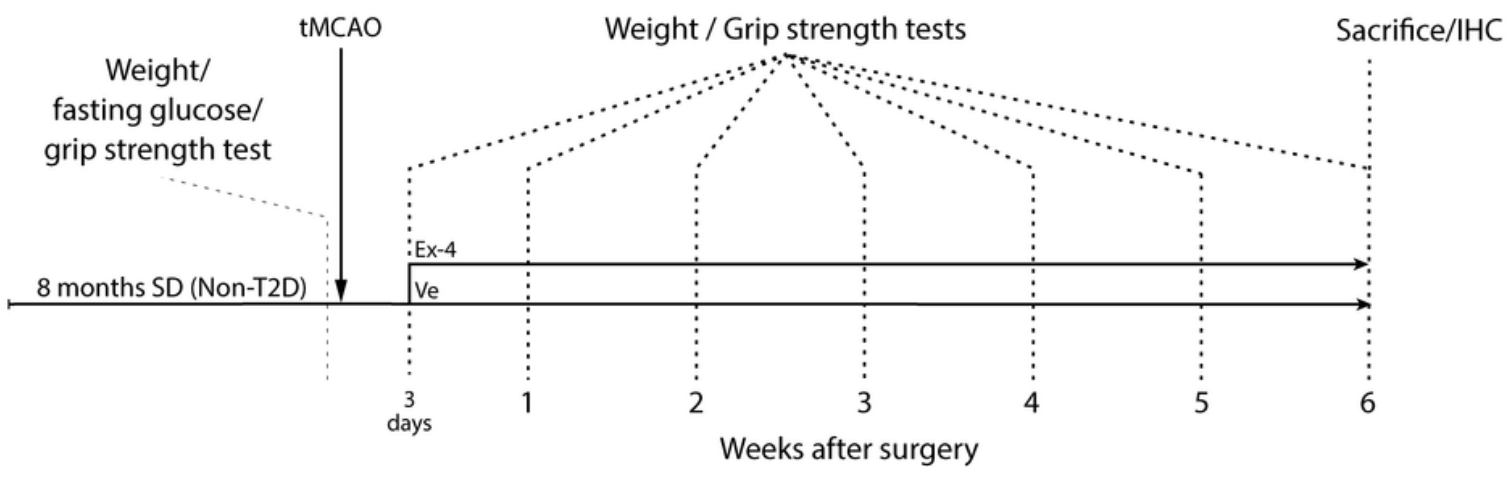

\section{Figure 1}

The experimental design. Experimental design of a Diabetic study and b Non-diabetic study. 
Diabetic study

Non-T2D $\square$ T2D-Ve $\square$ T2D-Ex-4

* = Non-T2D vs. T2D-Ve \#= T2D-Ve vs. T2D-Ex-4 \$ = Non-T2D vs. T2D-Ex-4

a

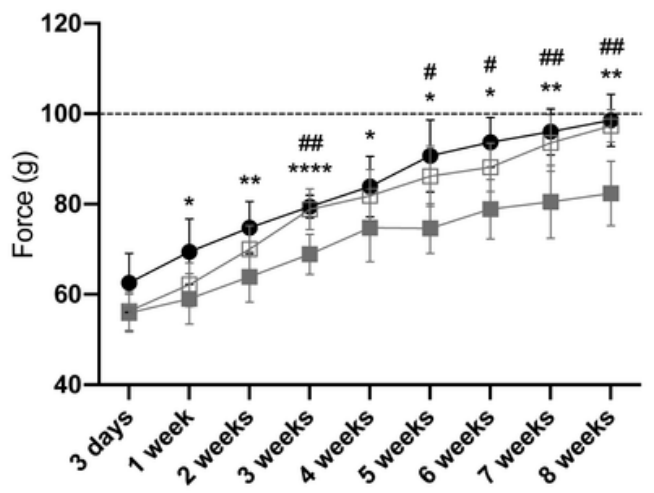

\begin{tabular}{|l|c|c|c|}
\hline Two-way Repeated measures ANOVA & & & \\
\hline & & & \\
\hline Source of Variation (Non-T2D vs. T2D-Ve) & \% of total variation & P value & P value summary \\
\hline Interaction: Time x T2D & 1,44 & 0,0496 & $*$ \\
\hline Time & 58,97 & $<0,0001$ & $\cdots * *$ \\
\hline T2D & 20,37 & $<0,0001$ & $\cdots * *$ \\
\hline & & & \\
\hline Source of Variation (T2D-Ve vs. T2D-Ex-4) & $\%$ of total variation & P value & P value summary \\
\hline Interaction: Time x Exendin-4 & 2,83 & 0,0008 & $\cdots *$ \\
\hline Time & 71,55 & $<0,0001$ & $* * * *$ \\
\hline Exendin-4 & 10,22 & 0,0007 & $* * *$ \\
\hline & & & \\
\hline Source of Variation (Non-T2D vs. T2D-Ex-4) & $\%$ of total variation & P value & P value summary \\
\hline Interaction: Time x Exendin-4 & 0,648 & 0,2642 & ns \\
\hline Time & 81,9 & $<0,0001$ & $\cdots * * *$ \\
\hline Exendin-4 & 1,949 & 0,0894 & ns \\
\hline
\end{tabular}

b

C
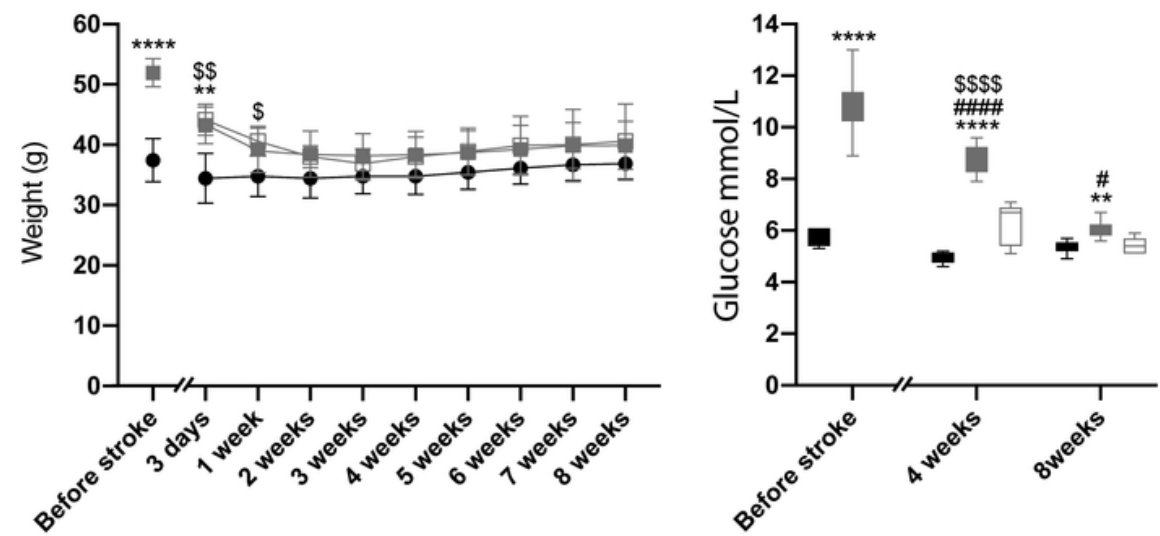

d

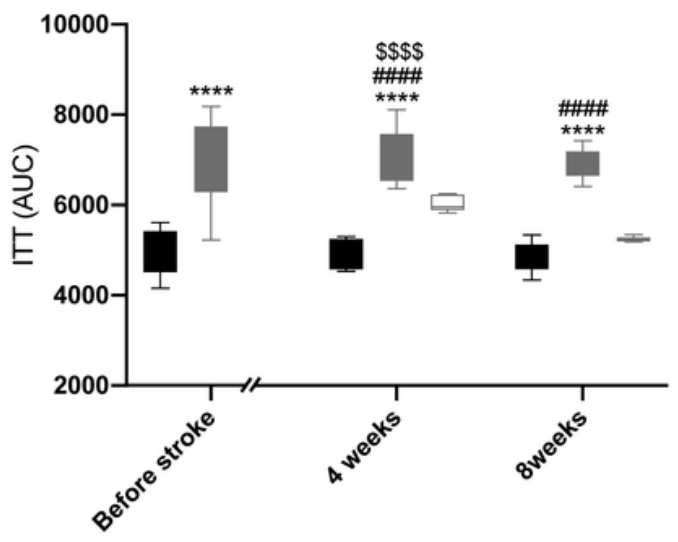

e

f
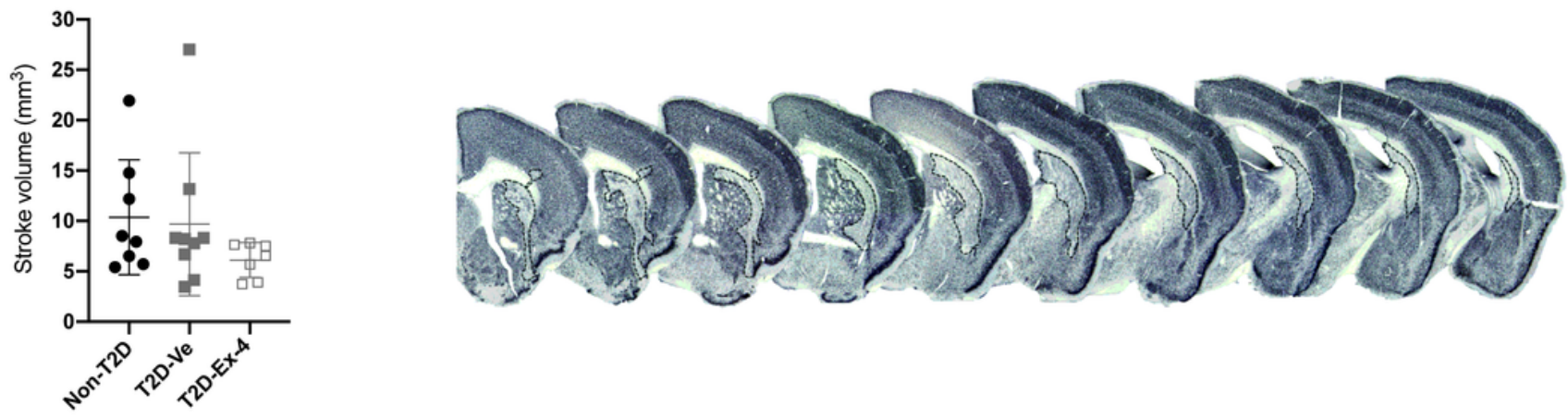

Figure 2

The effect of T2D and Exendin-4 treatment on neurological recovery, metabolic parameters and stroke volume after tMCAO. a Grip strength recovery and ANOVA table. Dashed line on a indicates mean of prestroke grip strength. b Body weight, c Fasting glucose, d Insulin sensitivity (insulin tolerance test, ITT) and e Stroke volume. $f$ Representative images of NeuN staining. The dotted lines on images on $f$ panel indicate stroke area. Data on $a, b$ and e are presented as mean $\pm S D$. Box plots on $c$ and $d$ show min-max values. Two-way repeated measures ANOVA $(a-d)$ or One-way ANOVA (e) followed by Tukey's, Sidak's or 
Bonferroni's tests was used. * denotes $p<0.05$, ** denotes $p<0.01$, **** denotes $p<0.0001$, \# denotes $p$ $<0.05$, \#\# denotes $p<0.01$ and \#\#\# denotes $p<0.0001$, $\$$ denotes $p<0.05$,

$$
\text { denotesp }<0.01, \text { and }
$$

$\$ \$$ denotes $p<0.0001$
- Non-T2D
- T2D-Ve
$\square \quad$ T2D-Ex-4

a

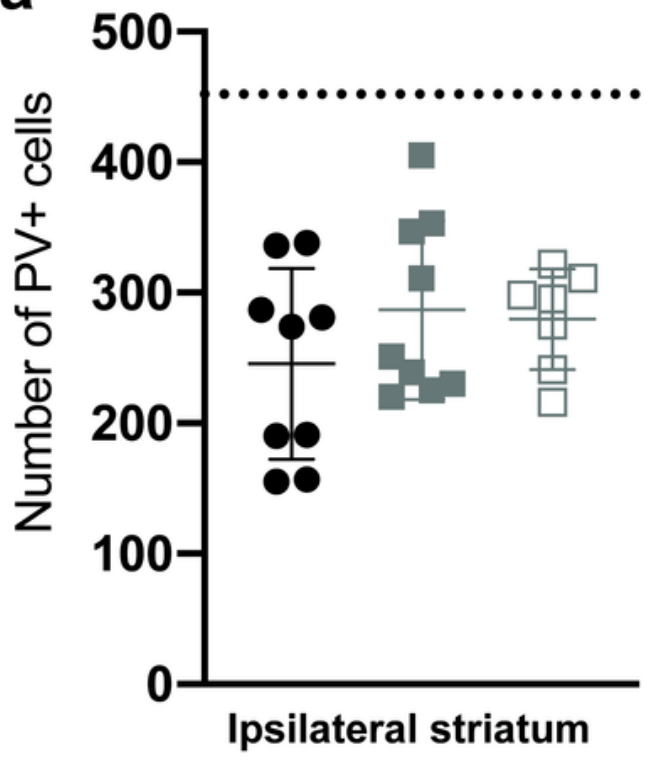

b

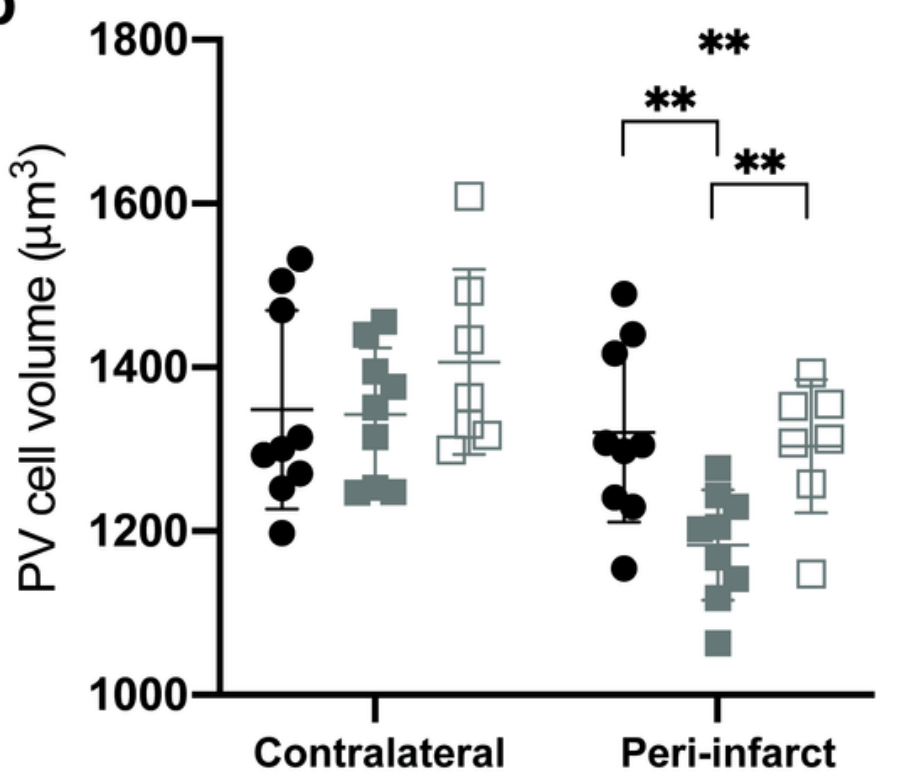

C

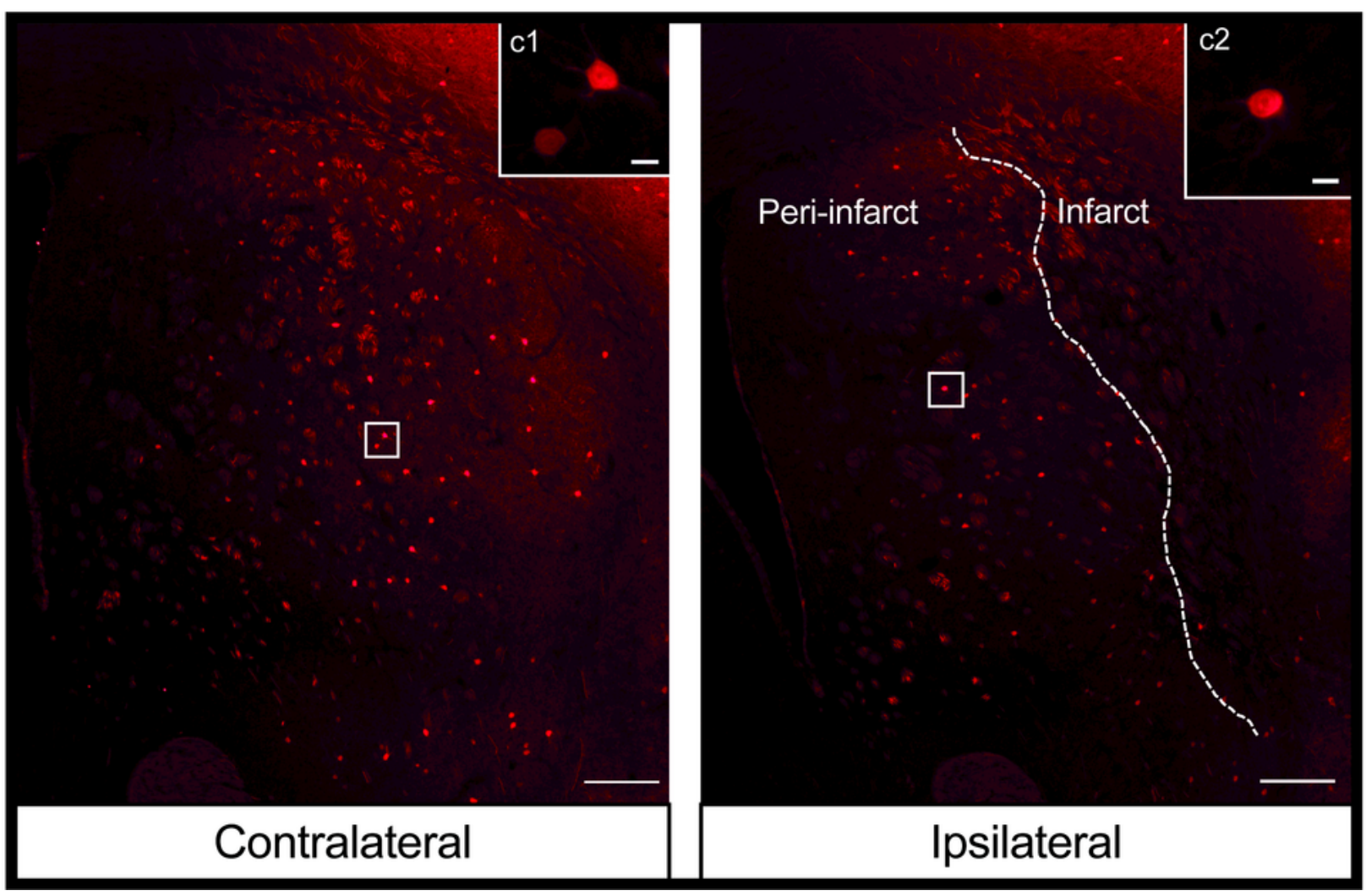

Figure 3 
Exendin- 4 counteracts the diet-induced atrophy of PV+ cells. a Number of PV+ cells in the contralateral (represented by dotted line) vs. ipsilateral striatum. b The average soma volume of PV+ cells. Data presented as mean $\pm S D$. Two-way ANOVA followed by Two-stage linear step-up procedure of Benjamini, Krieger and Yekutieli was used to compare the volume of PV+ cells between non-T2D vs. T2D-Ve vs. T2DEx-4. ${ }^{* *}$ denotes $p<0.01$. Floating asterisks (not attached to a line) indicate a difference in contralateral vs ipsilateral, in the same group. c Representative images of PV+ interneurons in contralateral and ipsilateral striatum, scale bars $=200 \mu \mathrm{m}$ and $25 \mu \mathrm{m}$ for inserts $\mathrm{c} 1$ and $\mathrm{c} 2$.

$\rightarrow$ Non-T2D

- T2D-Ve $\square$ T2D-Ex-4

a
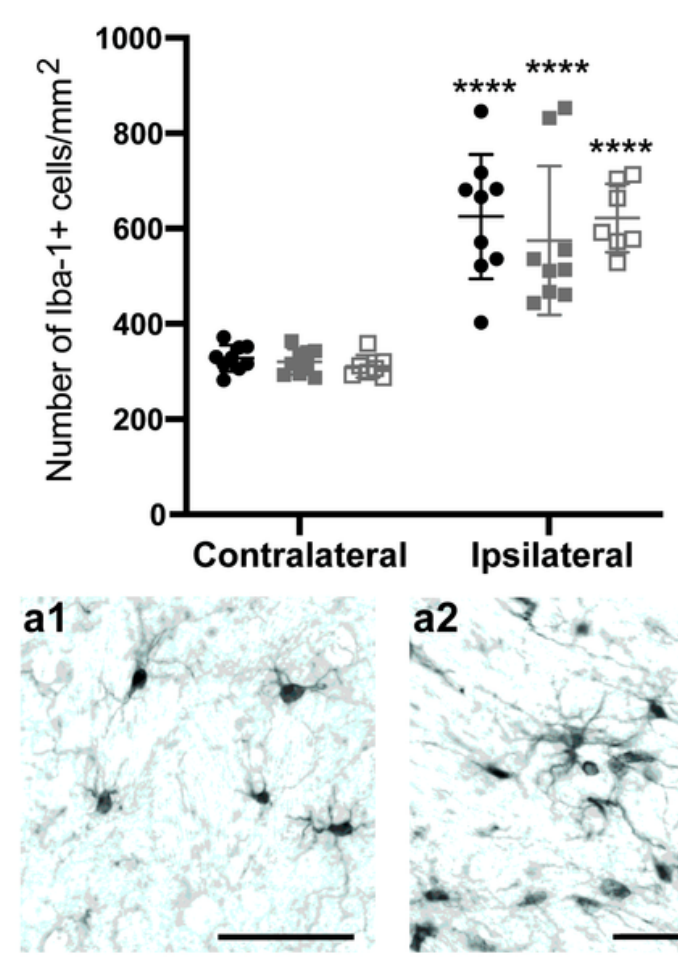

b
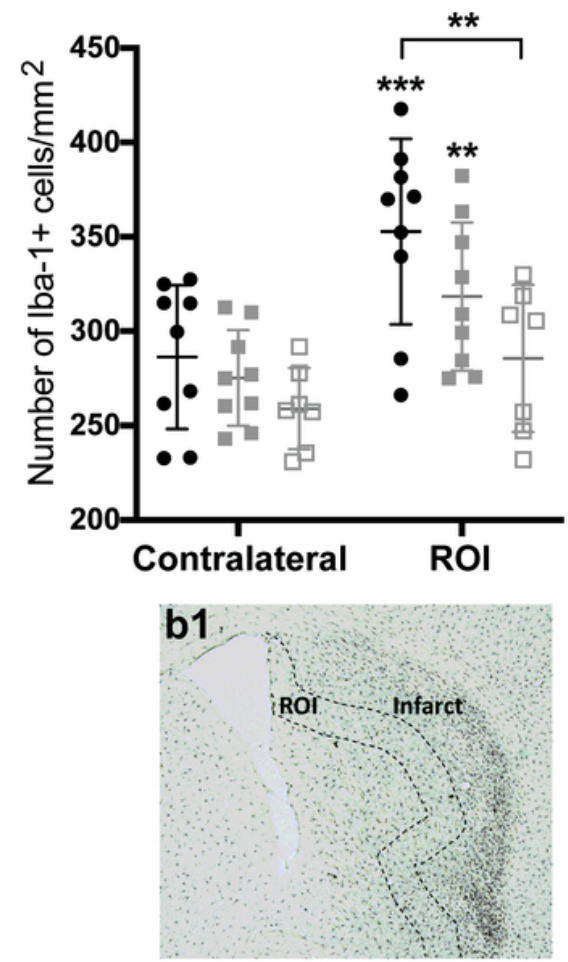

C
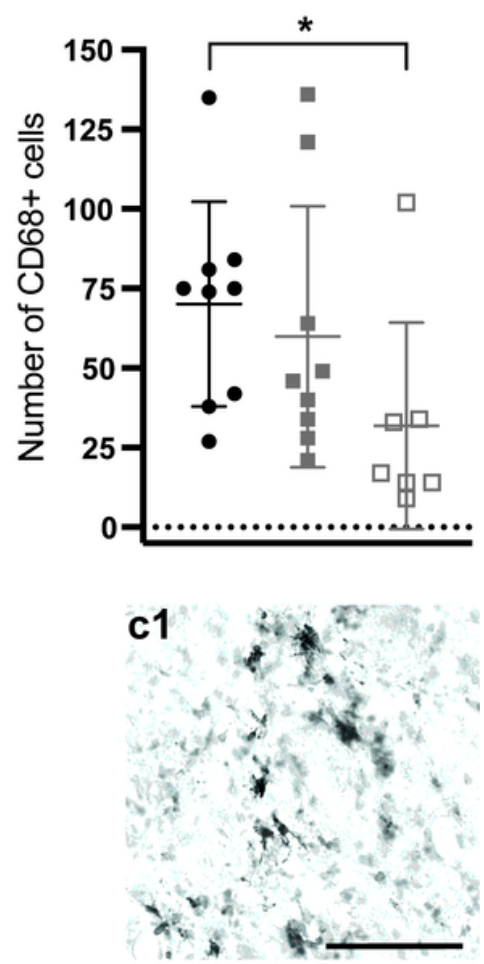

\section{Figure 4}

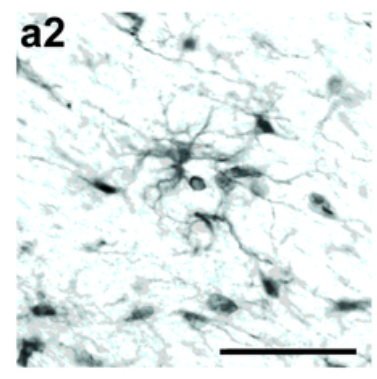

Exendin-4 decreases neuroinflammation after stroke. a Density of Iba- $1+$ cells at 8 weeks after stroke. Representative image of Iba-1+ cells in contralateral (a1) and ipsilateral striatum (a2). b Quantification of Iba-1+ cells in an infarct-adjacent region of interest (ROI). b1 Photomicrograph of Iba1 staining, ROI is outlined by dashed line. c Number of CD68+ cells in the ipsilateral striatum. c1 Representative image of $\mathrm{CD} 68+$ cells. Data are presented as mean $\pm S D$. Two-way ANOVA followed by Two-stage linear step-up procedure of Benjamini, Krieger and Yekutieli was used to compare the number of Iba-1+ cells in the contralateral vs. ipsilateral striatum (in a) and in contralateral vs. ROI (in b) between non-T2D vs. T2D-Ve vs. T2D-Ex-4. Kruskal-Wallis with uncorrected Dunn's test was used to compare the number of CD68+ 
cells between non-T2D vs. T2D-Ve vs. T2D-Ex-4. * denotes $p<0.05$, ** denotes $p<0.01$, *** denotes $p<$ 0.001 and $* \star \star \star$ denotes $p<0.0001$. Floating asterisks (not attached to a line) indicate a difference in contralateral vs ipsilateral, in the same group. Scale bars in a1, a2, and c1 = $50 \mu \mathrm{m}$.

a

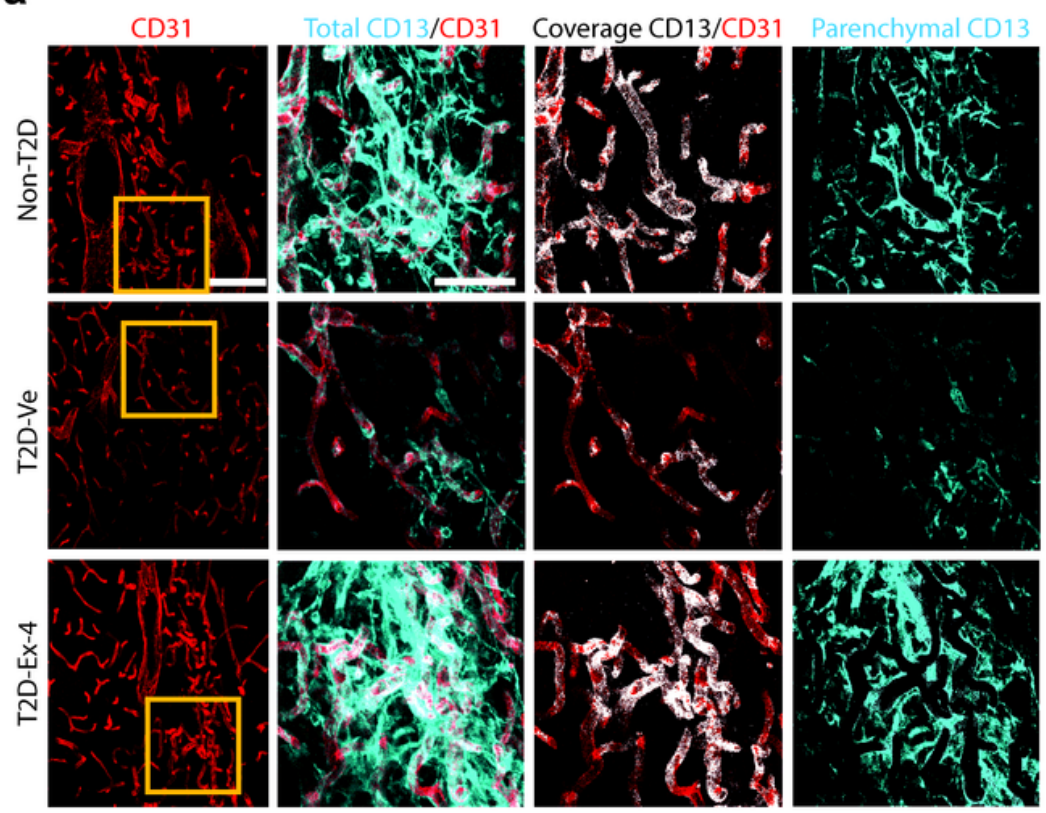

- Non-T2D

b

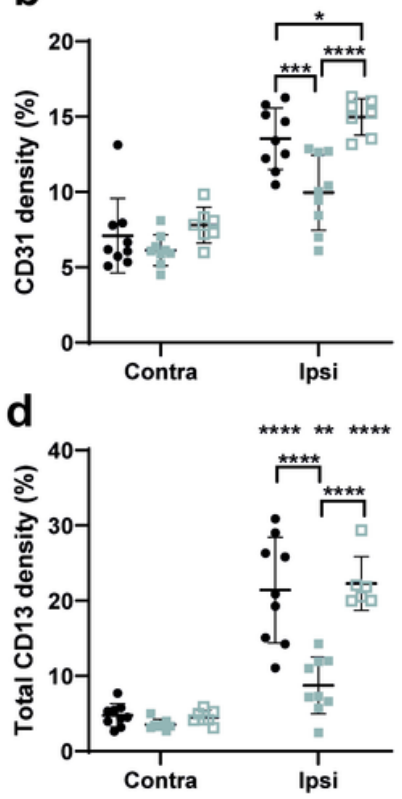

T2D-Ve T2D-Ex-4

C
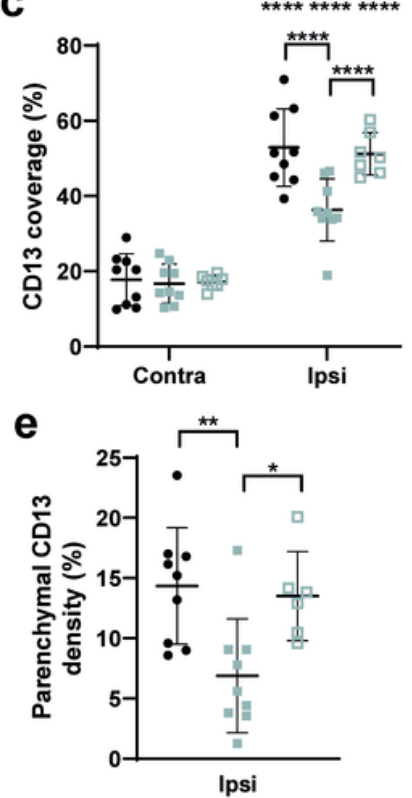

Figure 5

Exendin-4 restores the density and maturity of vessels in T2D mice after stroke. a Representative images of CD31+ (vessels) and CD13+ (pericytes) staining in the ipsilateral striatum. Representative images of the contralateral striatum are included in Additional file 1: Figure S2. Scale bar: $100 \mu \mathrm{m}$ for the left panel (CD31); $50 \mu \mathrm{m}$ for the two center and right panels (higher magnification). b Density of CD31+ vessels at 8 weeks after stroke. c Coverage of vessels by pericytes. d Total density of CD13+ pericytes, including both perivascular and parenchymal pericytes. e Density of parenchymal CD13+ pericytes. Data presented as mean $\pm S D$. Two-way ANOVA followed by Two-stage linear step-up procedure of Benjamini, Krieger and Yekutieli was used to compare the density of CD31+ vessels, the density of CD13+ pericytes, and coverage of CD31+ vessels by CD13+ pericytes, between non-T2D vs. T2D-Ve vs. T2D-Ex-4, in the contralateral and ipsilateral striatum. One-way ANOVA with Tukey post-hoc test was used to analyze the density of parenchymal CD13+ pericytes, in the ipsilateral striatum. * denotes $p<0.05$, ** denotes $p<$ $0.01, * \star \star$ denotes $p<0.001$ and $\star \star \star \star *$ denotes $p<0.0001$. Floating asterisks (not attached to a line) indicate a difference in contralateral vs ipsilateral, in the same group. 
Non-diabetic study

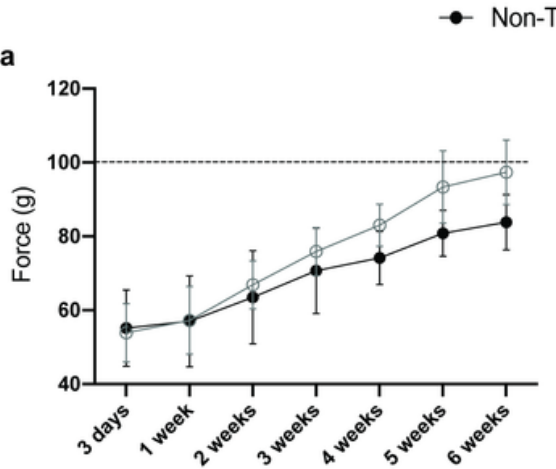

a

b
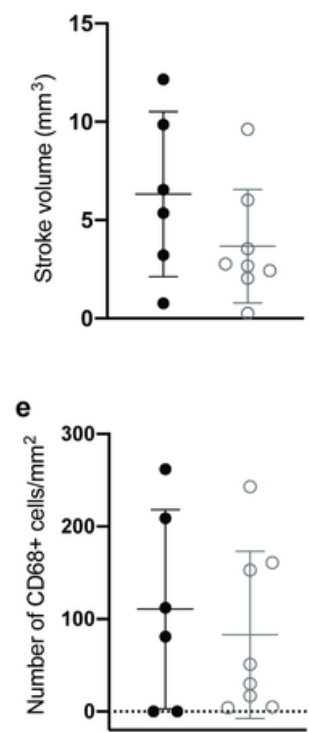

h

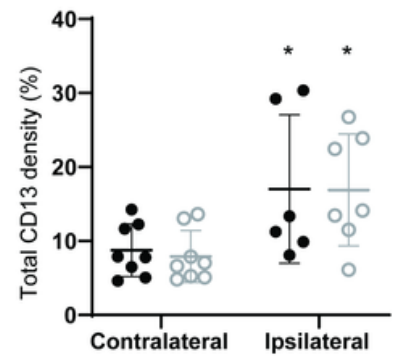

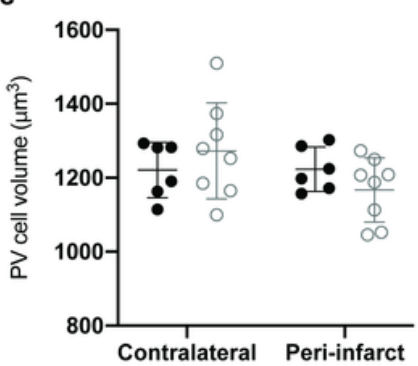

$\mathbf{f}$

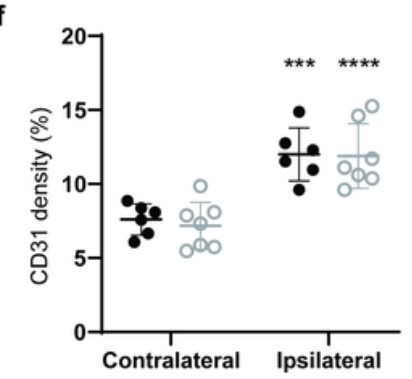

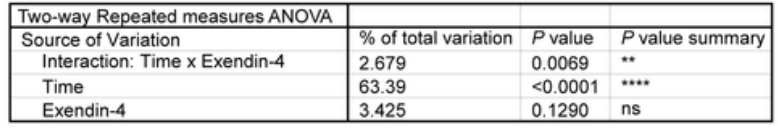

d

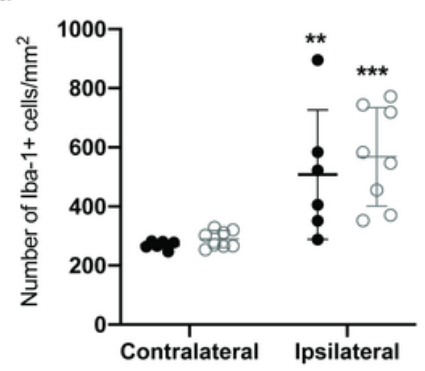

g

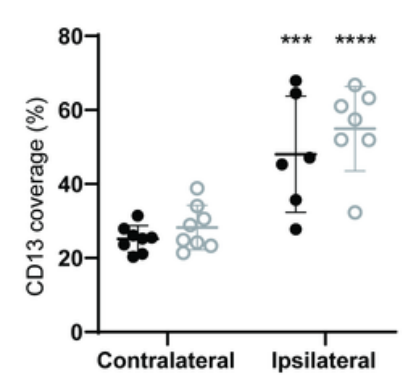

Figure 6

The effect of Exendin-4 treatment after $\mathrm{tMCAO}$ in non-diabetic mice. a Recovery of grip strength and ANOVA table. b Stroke volume. c Average volume of PV+ interneurons. d Number of Iba- $1+$ cells. e Number of CD68+ cells. $f$ Density of CD31+ vessels. $g$ Coverage of vessels by pericytes. $h$ Total density of CD13+ pericytes (including both perivascular and parenchymal pericytes). i Serum insulin levels. Data are presented as mean $\pm S D$. Two-way repeated measures ANOVA followed by Tukey's or Bonferroni's test was used to compare grip strength between non-T2D-Ve vs. non-T2D-Ex-4. Two-way ANOVA followed by Twostage linear step-up procedure of Benjamini, Krieger and Yekutieli was used to compare the volume of $\mathrm{PV}+$ cells, the number of Iba- $1+$ cells, the density of CD31+ vessels, the density of CD13+ pericytes, and 
coverage of CD31+ vessels by CD13+ pericytes. Unpaired t-test was used to analyze stroke volume,

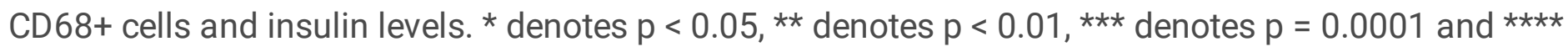
denotes $p<0.0001$. Floating asterisks (not attached to a line) indicate a difference in contralateral vs ipsilateral, in the same group.

\section{Supplementary Files}

This is a list of supplementary files associated with this preprint. Click to download.

- Additionalfile1.pdf 